\title{
COMPARISON OF TWO NANOFILTRATION MEMBRANE REACTORS FOR A MODEL REACTION OF OLEFIN METATHESIS ACHIEVED IN TOLUENE
}

\author{
Murielle RABILLER-BAUDRY ${ }^{1}{ }^{*}$, Ghassan NASSER ${ }^{1}$, Thierry RENOUARD ${ }^{1}$, \\ David DELAUNAY ${ }^{1,2}$, Martin CAMUS ${ }^{1}$
}

1- Université Rennes 1, UMR-CNRS 6226 «Institut des Sciences Chimiques de Rennes », 263 avenue du Général Leclerc, CS 74205, case 1011, 35042 Rennes cedex, France

2- present address : Université Caen-IUT Caen - Equipe de Recherche en Physico-Chimie et Biotechnologie EA 3914, Boulevard Maréchal Juin, 14032 Caen cedex, France

corresponding author : murielle.rabiller-baudry@univ-rennes1.fr

\begin{abstract}
The recent commercialisation of nanofiltration membranes resistant toward organic solvents is a real opportunity for fine chemistry. This study deals with different ways of integration of organic solvent nanofiltration for a specific type of reactions known as olefin metathesis and shows the use of two nanofiltration membrane reactors both running in cross-flow filtration mode $\left(0.1 \mathrm{~m} . \mathrm{s}^{-1}\right)$. They are used either in semi-continuous or continuous mode. A model ring closing metathesis reaction is chosen for the demonstration. A commercially available precatalyst, namely Grubbs-Hoveyda II, as well as a commercial polyimide membrane (Starmem 122, MWCO 220 g.mol ${ }^{-1}$ ) are used. Firstly, nanofiltration of the single pre-catalyst allows establishing that the highest retention is obtained with a 40 bar pressure. Secondly, this pressure is used with the two membrane reactors before testing other conditions. Finally, interests and limitations of the two reactors are discussed.
\end{abstract}

\section{KEYWORDS:}

Organic solvent nanofiltration; membrane reactor; olefin metathesis; organo-metallic catalyst; Grubbs-Hoveyda II pre-catalyst

\section{Introduction}

Nanofiltration has been first proposed at the end of the 80s for applications in aqueous media [1]. Thanks to its success at industrial scale and together with the development of chemically stable polymeric membranes, non-aqueous applications have progressively emerged and since the early 2000s, nanofiltration in organic solvent (OSN or SRNF) using polydimethylsiloxane (PDMS) or polyimide (PI) based membranes has also been developed. Industrial applications of OSN already exist, mainly for solvent recycling. For instance, the toluene/methylethylketone (MEK) separation in the Max-Dewax process (Beaumont, Texas, USA) is performed with PI membranes to recycle toluene [2]. Moreover, in this particular case, OSN increases the sustainability of the overall process because it allows a $20 \%$ decrease in energy consumption and a $25 \%$ increase in oil recovery [3]. Besides the petroleum industry, the pharmaceutical industry also uses OSN, but for extraction and/or purification of Active 
Pharmaceutical Ingredients (APIs). Once again, membranes are based on PI or PDMS [4-11] and, in most cases, solvents are mixtures made of water and organic polar solvents, such as alcohols. At laboratory scale, part of the initial driving force for the development of OSN has been the recovery of components of high added value. Among them are homogeneous (soluble) organo-metallic catalysts because both the metallic centre and the grafted ligands are quite expensive. In parallel to the recovery, the recycling of long term stable catalysts has also been studied [12-18].

Among organic reactions that are catalysed by homogeneous organo-metallic catalysts is the series of olefin metathesis reactions that provide access to many intermediates of high interest for fine chemistry. The olefin metathesis reaction, commonly achieved in toluene or dichloromethane, corresponds to the creation of a carbon-carbon double bond, either by interor intra-molecular rearrangement [19]. Classical homogeneous organo-metallic catalysts used are mainly based on ruthenium and those belonging to the Grubbs-Hoveyda II type are particularly stable. Several studies have been performed in order to optimise their life cycle and many routes have been proposed to recover these catalysts in order to reuse them in subsequent olefin metathesis cycles. For instance, the immobilization of the catalysts has been reported in non-conventional solvents [20] such as ionic liquids [21-23], supercritical fluids $[\mathbf{2 4}, \mathbf{2 5}]$ or perfluoroalkanes [26]. However, an important drawback of these methods was a rather difficult industrial scale-up. An alternative process to these physico-chemical approaches would be the use of membrane separation. The nanofiltration of olefin metathesis catalysts has already been reported [27-30]. In our group, OSN has been successfully used for the recovery of a set of Grubbs-Hoveyda II prototype catalysts in dead-end filtration mode with a Starmem 228 membrane (MET-Evonik, no more commercially available); up to 5 consecutive cycles of a model reaction of ring closing metathesis (RCM) have been achieved at room temperature in toluene or dimethylcarbonate (a green solvent) with a single load of catalyst [31]. On a fundamental point of view, the strategy followed in this previous study, as well as in all studies based on the recycling of olefin metathesis catalysts, was based on the current knowledge available at that time, in other words on the "release-return mechanism" also known as the "boomerang" effect. For sake of clarity, and because it is important to understand the study presented in this paper, this mechanism is quickly presented here in the case of the Grubbs-Hoveyda II catalyst (Figure 1). It was currently admitted for all catalysts belonging to this type until 2010. Because the organo-metallic catalyst is not sufficiently stable to be long-time stored, it is introduced in the reaction medium as a stable form called "pre-catalyst". In presence of the substrate to be transformed, the pre-catalyst evolves in its active form by the isopropoxystyrene ligand release. At the end of the reaction the product is delivered and the isopropoxystyrene ligand returns on the active form of the catalyst to reform the pre-catalyst entity; this final step is known as the "boomerang effect'. The assumption of the release-return mechanism is compatible with the concept of the pre-catalyst recyclability.

Looking for an in-situ sensor to follow the olefin metathesis reaction during OSN, we started to systematically record UV-Vis spectra of the reaction media containing the GrubbsHoveyda II pre-catalyst (together with the substrate and the product but both of them being transparent in the chosen UV-Vis range at the chosen concentrations). At the start of the reaction, the registered spectrum corresponded in fact to that of the pure pre-catalyst with a maximum absorption located at $377 \mathrm{~nm}$ (see results for details). But, the spectrum evolved during the reaction, regardless of the pre-catalyst used. The band at $377 \mathrm{~nm}$ decreased but did not reappear at the end of the reaction. Accordingly, the pre-catalyst seemed definitely destroyed. 
At the same period, Vorfalt et al. [32] achieved an in-depth study of the olefin metathesis mechanism, initially based on the same observation of the disappearance of the maximum absorption band in UV-Vis. They have tagged the isopropoxystyrene ligand with a specific fluorescent group: when the tagged ligand was free the fluorescence existed whereas when the ligand was coordinated to the metal centre then the fluorescence did not exist anymore. They have followed the evolution of the fluorescence spectrum of the reaction medium of a model reaction; it allowed these authors to deny the "boomerang effect" hypothesis: the final step of the isopropoxystyrene ligand return did not exist.

From this conclusion, the concept of the pre-catalyst recycling falls down in the case of olefin metathesis. Consequently another concept must be proposed to improve the catalyst life cycle. In other words, in presence of a substrate, the pre-catalyst must be considered as a stable reservoir evolving in the "unstable" active catalyst form. The percentage of pre-catalyst transformed in its active form is not known. At the end of the metathesis reaction that involves an unknown number of substrate molecules per catalyst molecule, a deactivated form containing $\mathrm{Ru}$ is obtained (not clearly identified nowadays). Of course, this deactivated form exhibits no more catalytic activity.

In this context, the objective of this paper was to study how organic solvent nanofiltration could contribute to the optimisation of the metathesis reaction. We first focused the experimental study on the understanding of the pre-catalyst behavior in the reaction medium. In order to find the best possible performances, the olefin metathesis was performed either in a semi-continuous membrane reactor or in a continuous one. For this second reactor, the aim was to decrease the residence time of the active catalyst without any substrate because this situation would lead to its fast deactivation. For this purpose a model reaction of ring closing metathesis (RCM) was chosen; this reaction has commonly been used by chemists to test the activity of new pre-catalyst prototypes but has no industrial application [33]. Nevertheless, in the future, results could be re-investigated for reactions performed on bio-resources for added value applications.

\section{Experimental}

\subsection{Catalysis and analytical tools}

\subsubsection{Pre-catalysts and their analyses}

In this paper, the Grubbs-Hoveyda II pre-catalyst (Fig. 2, pre-catalyst 1) was mainly used. It was provided by Sigma-Aldrich.

Several other pre-catalysts (Fig. 2, pre-catalysts 2-4) were punctually used for sake of comparison. These prototypes have been specially synthesised for this study by S. Shahane et al. [34]. They were slightly modified when compared with the initial Grubbs-Hoveyda II structure. To enhance the retention of the active catalysts deriving from these new structures, the modifications were applied on the $N$-heterocyclic carbene part which always remained coordinated to the ruthenium. It has been checked that the activity of these pre-catalysts was close to that of the un-modified Grubbs-Hoveyda II in the model ring closing metathesis of diethyl diallyl malonate (DEDAM). To avoid degradation due to oxygen all pre-catalysts were stored under argon. 
UV-Vis spectra of each pre-catalyst in toluene were recorded in the $290-450 \mathrm{~nm}$ range with a spectrometer provided by Uvikon and equipped with a quartz tube of $1 \mathrm{~cm}$ thickness. The background was recorded with pure toluene. Thanks to the high absorbance of the $0.5 \cdot 10^{-3}$ $1.0 \cdot 10^{-3}$ mol. $\mathrm{L}^{-1}$ pre-catalyst solutions, an appropriate dilution with toluene was performed prior to the spectrum record. The objective was to have a maximum absorbance lower than one absorbance unit. Then, for sake of simplification for the comparison, all experimental spectra were magnified by the dilution factor and the UV-Vis spectra shown in this paper correspond to recalculated ones including this dilution correction.

During OSN of the single Grubbs-Hoveyda II pre-catalyst in toluene, the profile of the UVVis spectra did not varied. Thus the concentration of the pure pre-catalyst was estimated from its maximum absorbance at $\lambda_{\max }=377 \pm 1 \mathrm{~nm}$ using the Beer-Lambert relationship (it was checked that all studied pre-catalysts had the same maximum absorbance wavelength).

Neither DEDAM nor c-DEDAM (at the concentrations of the experiments) absorbed the UVVis light in the 290-450 nm range. Consequently, in the course of the RCM reactions the UVVis spectra revealed only the behavior of the pre-catalyst. Thus comparison of spectra over time can be used to control/follow any change in the pre-catalyst structure, and probably could reveal some of the entities issued from the pre-catalyst, namely active and de-activated forms.

\subsubsection{Reagents and their analyses}

Toluene of analytical grade was provided by Sigma-Aldrich. It was used as received for the determination of the reference membrane flux and also for the rinsing steps. But to avoid any water and oxygen traces, toluene was distilled over sodium prior its use in metathesis reactions or before OSN of the single pre-catalysts.

DiEthyl DiAllyl Malonate of technical grade was provided by Acros. Gas chromatography of DEDAM revealed the presence of several un-identified impurities for an overall amount lower than $1 \%$ (not shown). These impurities remained unchanged during the metathesis reactions, regardless of the pre-catalyst involved. Thus no more comments will be added about them in the following text.

DEDAM and c-DEDAM concentrations were determined from gas chromatography (GC) quantification with a Shimadzu GC gas chromatograph (GC-2014). The semi-capillary column used was provided by Phenomenex (Equity $^{\mathrm{TM}}-5,30 \mathrm{~m} \times 0.53 \mathrm{~mm} \times 1.5 \mu \mathrm{m}$ film thickness). This column was mainly apolar thanks to the stationary phase made of PDMSPDPS (95-5). Injection achieved at $250{ }^{\circ} \mathrm{C}$ was followed by analysis at a constant temperature set at $230{ }^{\circ} \mathrm{C}$. The detection was obtained by a FID detector set at $250{ }^{\circ} \mathrm{C}$. The carrier gas was nitrogen at $18 \mathrm{kPa}$. Quantification by the internal standard method was obtained with an accuracy of $1 \%$.

\subsubsection{Olefin metathesis reaction and its evaluation}

Figure 3 shows the model Ring Closing Metathesis (RCM) reaction. It was performed at room temperature. DEDAM was transformed in a cyclopentene form (c-DEDAM). The DEDAM to pre-catalyst ratio at the reaction start varied from 100 to 200 (see below). 
The RCM performances were first evaluated by the DEDAM conversion rate $\left(\mathrm{Tau}_{\mathrm{t}}\right)$ at a given time $(\mathrm{t})$ according to equation 1. The final (maximum) conversion rate was called Tau. When conversion was greater than $97 \%$, accuracy on conversion was $3 \%$.

$$
\mathrm{Tau}_{\mathrm{t}}=\mathrm{c}-\mathrm{DEDAM}_{\mathrm{t}} / \mathrm{DEDAM}_{\mathrm{i}}
$$

With:

DEDAM $_{\mathrm{i}}$ the initial mole number of DEDAM.

c-DEDAM the mole number of c-DEDAM at the given $t$ time.

The purity of obtained c-DEDAM was evaluated by equation 2 (the Ru traces were neglected for this first evaluation).

$$
\text { Purity = c-DEDAM / (c-DEDAM + DEDAM })
$$

With:

Purity: the c-DEDAM purity in the permeate

DEDAM the mole number of DEDAM in the final medium

c-DEDAM the mole number of c-DEDAM in the final medium

Considering the catalyst performance, the apparent turnover number $\left(\mathrm{TON}_{\mathrm{t}}\right.$,apparent $)$ at a given time (t) was determined from the initial load of pre-catalyst, according to equation 3. The cumulative value at the end of the cascade of several RCM reactions was further called TON.

$\mathrm{TON}_{\mathrm{t}, \text { apparent }}=\left(\mathrm{c}-\mathrm{DEDAM}_{\mathrm{t}}\right) /($ initial pre-catalyst $)$

With:

$\mathrm{c}-\mathrm{DEDAM} \mathrm{T}_{\mathrm{t}}$ : the overall mole number of formed c-DEDAM at $\mathrm{t}$ time.

initial pre-catalyst: the mole number loaded at the first RCM cycle ("RCM cycle 1 start") for a cascade of 1 to 4 consecutives RCM cycles.

\subsection{Membrane and nanofiltration experimental set up}

\subsubsection{Membrane}

The Starmem 122 membrane was provided by MET-Evonik (London, U.K.). It was an integrally skinned and asymmetric membrane with a polyimide active layer. Its molecular weight cut-off was $\mathrm{MWCO}=220 \mathrm{~g} \cdot \mathrm{mol}^{-1}$ (according to the manufacturer and obtained from OSN of n-alkanes dissolved at $2 \mathrm{wt} \%$ in toluene). Membrane disks with a filtering area of 17 $\mathrm{cm}^{2}$ were cut in a flat membrane sheet. Prior to OSN, the pristine membrane was rinsed by toluene in order to remove the preservatives. Then the membrane was compacted in toluene at room temperature under 40 bar until a constant flux was reached. This flux was further called the "reference flux" $\left(\mathrm{J}_{0}\right.$, at $\left.26 \pm 3^{\circ} \mathrm{C}\right)$. The membrane permeability to toluene $\left(\mathrm{Lp}_{0}\right)$ was determined according to the well-known Darcy's law:

$$
\mathrm{J}_{0}=\mathrm{Lp}_{0} \times \mathrm{TMP}
$$

With:

TMP : the applied transmembrane pressure 
It can be noticed that a Duramem 300 membrane (MET-Evonik, London, UK) had been tested prior to the Starmem 122 selection. Its molecular weight cut-off was MWCO $=300 \mathrm{~g} \cdot \mathrm{mol}^{-1}$ (according to the manufacturer and obtained from OSN of styrene oligomers dissolved at $2 \mathrm{wt}$ $\%$ in acetone). The Duramem 300 flux in toluene was ten times lower than that measured with the Starmem 122. Moreover the retention of the Grubbs-Hoveyda II pre-catalyst $\left(0.5 \times 10^{-3}\right.$ mol. $\mathrm{L}^{-1}$ ) was not sufficient, ranging from $88 \%$ to $46 \%$ when OSN was performed between 10 and 40 bar in dead-end mode, respectively.

\subsubsection{OSN of single Grubbs-Hoveyda II pre-catalyst}

OSN was performed at $25^{\circ} \mathrm{C}$ in a cross-flow mode using the MET-Cell equipment provided by MET-Evonik (London, UK) (Figure 4). In standard conditions, the initial feed volume was $\mathrm{V}_{\mathrm{i}}=300 \mathrm{~mL}$, the cross flow velocity was $0.1 \mathrm{~m} \cdot \mathrm{s}^{-1}$ obtained thanks to the pump on the retentate circuit. TMP varied in the range 10-40 bar thanks to the nitrogen pressure applied on the feed tank. Permeate was continuously extracted.

OSN was also performed at $25^{\circ} \mathrm{C}$ in dead-end mode using the MET-Cell equipment after the removal of the pump. In standard conditions, the initial feed volume was $\mathrm{V}_{\mathrm{i}}=300 \mathrm{~mL}$. TMP varied in the range 10-40 bar and permeate was also continuously extracted.

Regardless of the filtration mode, OSN performances were evaluated from both the membrane flux evolution and the solute retention. The flux measured during OSN of single solutes or mixtures was called $\mathrm{J}_{\mathrm{OSN}}$. Accuracy on flux measurements was $2 \%$. Because of the continuous extraction of the permeate, both in cross-flow and dead-end mode, the volume remaining in the feed tank decreased with the filtration time. Accordingly, the volume reduction ratio (VRR) increased (Equation 5). The maximum $V_{R R}$ was limited to VRR = $3.0 \pm 0.2$ due to the dead volume of the equipment.

$$
V R R_{t}=\frac{V_{i}}{V_{t}}=\frac{V_{i}}{V_{i}-\Sigma V_{p}}
$$

With:

$\mathrm{VRR}_{\mathrm{t}}$ the volume reduction ratio reached at a given time $(\mathrm{t})$

$\mathrm{V}_{\mathrm{i}}$ : the initial volume in the cell ( $300 \mathrm{~mL}$ in standard conditions).

$\sum \mathrm{V}_{\mathrm{p}}$ : overall extracted permeate volume at $\mathrm{t}$ time.

The flux measured during cross-flow OSN of the single Grubbs-Hoveyda II pre-catalyst (1.0 and $0.5 \times 10^{-3}$ mol. $\mathrm{L}^{-1}$ ) was almost equivalent to $\mathrm{J}_{0}$ regardless of the TMP in the range 10-40 bar. It can be drawn that no significant fouling occurred. Similar comments can be written for OSN of the other pre-catalysts $2-4$ at $0.5 \times 10^{-3}$ mol. $\mathrm{L}^{-1}$. Nevertheless the membrane was carrefully rinsed by toluene between each filtration experiment.

For dead-end OSN of the single Grubbs-Hoveyda II pre-catalyst $\left(0.5 \times 10^{-3} \mathrm{~mol}^{-1}\right)$, $\mathrm{J}_{\mathrm{OSN}}$ was always lower than $\mathrm{J}_{0}$. Consequently the membrane was carrefully rinsed by toluene. This simple treatment allowed the recovery of the initial permeate flux to toluene.

Observed retentions of pre-catalysts were calculated according to Equation 6 with a precision of $3 \%$.

$$
\text { Ret }=1-\mathrm{C}_{\mathrm{p}} / \mathrm{C}_{\mathrm{r}}
$$


With:

$\mathrm{C}_{\mathrm{p}}$ and $\mathrm{C}_{\mathrm{r}}$ the concentration of the solute in permeate and retentate, respectively, obtained from UV-Vis quantification.

\subsection{Membrane reactor for semi-continuous olefin metathesis reactions}

This membrane reactor had been used with all pre-catalysts depicted in Figure 2.

The OSN loop shown in Figure 4 was used as membrane reactor running in a semicontinuous mode. The feed cell was first filled with the appropriate pre-catalyst amount (200 $\mathrm{mg}, 3.2 \times 10^{-4} \mathrm{~mol}$ ) dissolved in less than $300 \mathrm{~mL}$ toluene and the solution was carrefully flushed by nitrogen in order to avoid any oxigen entrance that could limit the RCM reaction. Then a first load of DEDAM $(7.7 \mathrm{~g}, 0.032 \mathrm{~mol})$ dissolved in a small volume of toluene was introduced in the cell. Finally, the overall volume of toluene was $300 \mathrm{~mL}$, corresponding to $10^{-3}$ mol. $\mathrm{L}^{-1}$ pre-catalyst concentration and 0.106 mol. $\mathrm{L}^{-1}$ DEDAM concentration in the membrane reactor.

The RCM reaction (Figure 3) took place directly in the feed tank of the MET-Cell equipement at $31 \pm 3^{\circ} \mathrm{C}$ under a TMP of 1 bar adjusted by nitrogen. This TMP was too low to allow permeation through the membrane, but maintained an inerte atmosphere during all the RCM reaction. During RCM, the reaction mixture circulated at a $0.1 \mathrm{~m} . \mathrm{s}^{-1}$ cross-flow velocity in the OSN loop thanks to the pump installed on the retentate circuit. When the maximum conversion of DEDAM was obtained (controlled by gas chromatography on retentate samples), a 40 bar TMP was applied and permeate was continuously extracted. The 40 bar TMP was maintained up to the maximum reachable VRR $\approx 3.0$. This first step of the overall cascade corresponded to the so-called "RCM cycle $1+$ OSN step 1". It finally led to two fractions corresponding to permeate 1 and retentate 1 of Figure 5, respectively.

After this first DEDAM load, several consecutive cycles were achieved without any new addition of pre-catalyst. Only a new load of $0.032 \mathrm{~mol}$ of DEDAM in $200 \mathrm{~mL}$ toluene was introduced in order to reach $300 \mathrm{~mL}$ at each RCM cycle start, and so on. Four sequences of "RCM cycle + OSN step" were achieved (Figure 5). Finally two diafiltration steps were performed by addition of $2 \times 200 \mathrm{~mL}$ toluene in order to enhance the recovery of c-DEDAM.

During each OSN step, permeate was regularly collected in order to determine the DEDAM and c-DEDAM retentions, with a precision of $2 \%(\mathrm{GC})$, as well as the pre-catalyst retention (UV-Vis). Moreover the UV-Vis spectrum of the retentate was recorded at the end of the RCM cycle and at the end of each OSN step (in order to evidence that OSN did not modify the spectrum profile).

During this cascade of "RCM cycle + OSN step", the membrane flux regularly decreased from one step to the other. At the end of the two diafiltration steps, the membrane was carrefully rinsed by toluene until $\mathrm{J}_{0}$ full recovery.

The same protocol was also achieved with an initial load of $1.6 \times 10^{-4} \mathrm{~mol}$ (in $300 \mathrm{~mL}$ : $0.50 \pm$ $0.0310^{-3}$ mol. $\mathrm{L}^{-1}$ ) of pre-catalysts $\mathbf{1 - 4}$ with reloads of 0.032 mol DEDAM. Due to the lower conversion rates obtained, only 3 (RCM cycle + OSN step) and a diafiltration step were realised with pre-catalysts $\mathbf{1 , 2 , 4}$; only 2 (RCM cycle + OSN step) and a final diafiltration step with the pre-catalyst 3. 


\subsection{Membrane reactor for continuous olefin metathesis reaction}

This membrane reactor has been used only with the Grubbs-Hoveyda II pre-catalyst.

Figure 6 shows the lab-made membrane reactor able to run in continuous mode. The circulation loop was inspired from that of the semi-continous membrane reactor: the same circular membrane cell was used as well as the same pump. The volume of the circulation loop was close to $60 \mathrm{~mL}$. This loop was fed by two tanks: feed tank 1 delivered pure toluene thanks to the HPLC pump 1 whereas feed tank 2 provided DEDAM dissolved in toluene thanks to the HPLC pump 2. TMP in the loop was mainly controlled by the toluene addition via HPLC pump 1 flow rate.

The RCM reaction was achieved with a single load of pre-catalyst $\left(1.6 \times 10^{-4} \mathrm{~mol}\right)$ in order to compare with the membrane reactor running in a semi-continuous mode that was detailed above.

First, the Grubbs-Hoveyda II pre-catalyst solution $(60 \mathrm{~mL})$ was introduced in the circulation loop. For sake of homogenization, the recirculation at $0.1 \mathrm{~m} . \mathrm{s}^{-1}$ was maintained few minutes at 40 bar. This TMP was chosen because the pre-catalyst was highly retained at this pressure (at least $99.5 \%$ ). In the following the pre-catalyst remained confined in the loop maintained at $32 \pm 4{ }^{\circ} \mathrm{C}$.

Feed tank 2 was filled by a solution of DEDAM $(0.032 \mathrm{~mol}$ in $40 \mathrm{~mL}$ toluene). All DEDAM was progressively added to the loop (see results). Thanks to the pre-catalyst localization, the RCM reaction takes place in the circulation loop at the chosen TMP, either 10 or 40 bar (see results). During RCM, permeate was continuously extracted and TMP was maintained to the target value by delivering a toluene + DEDAM volume exactly corresponding to the extracted permeate volume.

At the end of the experiment performed at $40 \mathrm{bar}$, the membrane was demounted after a careful rinsing step allowing the membrane flux recovery. Then the membrane was dried and analysed by SEM-EDX (micro-analysis coupled to scanning electron microscopy) in order to highlight its elemental composition. This analysis was achieved with a JSM 6400 microscope provided by Jeol and equipped with an EDX analyser (Oxford Instrument, UK) and the INCA software acquisitions were done during $50 \mathrm{~s}$ at $20 \mathrm{kV}$ (magnification: $\mathrm{x} 400$ ).

\section{Results and discussion}

\subsection{OSN of single Grubbs-Hoveyda II pre-catalyst}

The main objective of this preliminary experiment was to reach the highest retention of the pre-catalyst in order to determine the better conditions to keep it, as long as possible, in the filtration loop of the membrane reactors.

\subsubsection{Selection of the OSN operating mode}


OSN of the Grubbs-Hoveyda II pre-catalyst at $0.50 \pm 0.0310^{-3} \mathrm{~mol} . \mathrm{L}^{-1}$ was achieved by studying firstly the effect of the transmembrane pressure on its retention in both dead-end and cross-flow filtration modes. Several independent filtrations were performed at a given pressure up to the same final VRR. Figure 7 summarizes the retention at $\mathrm{VRR}_{\text {final }} \approx 3$ according to the TMP.

In dead-end mode, the membrane permeability decreased linearly with the OSN time at a given TMP. At the end, the final permeability to initial permeability ratio ( $\left(\mathrm{p}_{\text {final }} / \mathrm{Lp}_{\text {initial }}\right)$ varied from 0.06 to 0.35 depending on the applied TMP (Table 1). The flux reduction was fully reversible regardless of the TMP; thus it can be drawn that concentration polarisation was important in this filtration mode, especially at low TMP. Simultaneously the pre-catalyst retention at $\mathrm{VRR} \approx 3$ was equal to $91 \%$ when the TMP increased from 10 to 20 bar and then decreased to $86 \%$ when the TMP increased from 30 to 40 bar (Figure 7).

In cross-flow mode, during OSN of the pre-catalyst, the membrane permeability remained that measured in pure toluene, regardless of the TMP and of the VRR: no flux decrease appeared. Simultaneously, the pre-catalyst retention at VRR $\approx 3$ increased from $96.3 \%$ to 99.5\% when the TMP increased from 10 to 30 bar, respectively, and then remained constant up to 40 bar (Figure 7). According to the convection-diffusion model [35-37], the retention increase with TMP suggested a predominant role of diffusion compared to convection before the membrane entrance (even if polarisation concentration is not able to be evidenced easily here).

Finally the cross-flow mode, as expected, was the best one to ensure simultaneously a high retention and a high flux. In the following a TMP of 40 bar was selected.

\subsubsection{Effect of the pre-catalyst concentration in cross-flow mode at 40 bar}

OSN was performed at 40 bar in cross-flow mode with different initial pre-catalyst concentrations ranging from $0.05 \times 10^{-3}$ to $1.40 \times 10^{-3}$ mol. $\mathrm{L}^{-1}$. For each initial concentration, the pre-catalyst retention was measured during OSN (meaning with an increasing VRR). No flux reduction was observed for the overall set of experiments.

Figure 8 shows the retention plotted against the concentration in the feed that depends on OSN time and thus on $\mathrm{VRR}_{\mathrm{t}}$ (up to $\mathrm{VRR}_{\text {final }} \approx 3$ ). The pre-catalyst retention increased from $94.8 \%$ to $99.5 \%$ when the concentration increased from $0.05 \times 10^{-3} \mathrm{~mol}^{-1} \mathrm{~L}^{-1}$ to $3.00 \times 10^{-3}$ mol. $\mathrm{L}^{-1}$. Then the retention remained constant at $99.5 \%$ when the concentration increased up to $5.00 \times 10^{-3} \mathrm{~mol} . \mathrm{L}^{-1}$.

These results must be kept in mind when dealing with the diafiltration step at the end of RCM reactions performed in the membrane reactors.

\subsection{Retention of DEDAM and c-DEDAM in cross-flow mode at 40 bar.}

The objective of the RCM was, of course, to obtain the highest amount of c-DEDAM (product) from the DEDAM (substrate) transformation. To reach this goal, a high retention of DEDAM in the membrane reactor was a need together with a high conversion rate. From preliminary experiments, it seemed that both DEDAM and c-DEDAM retentions were not affected by the presence of the pre-catalyst. Moreover the DEDAM retention seemed not affected by the presence of c-DEDAM, and conversely (not shown). During OSN of both 
DEDAM and c-DEDAM, the membrane permeability significantly decreased. Two main phenomena could explain this observation: the membrane compaction [38] and an important concentration polarization. In our case, since the membrane sheet was in place for more than 2 years, frequently operated at 40 bar, the membrane compaction was assumed to be complete for long. Moreover, OSN of the same volumes of toluene or of the pre-catalyst in toluene solution revealed relatively limited flux reduction, always reversible. So polarization concentration was obvious.

At 40 bar, in cross-flow mode, the DEDAM retention depended on its concentration (Figure 9). The retention was only $90 \%$ with a $106 \times 10^{-3}$ mol. $\mathrm{L}^{-1}$ concentration which corresponded to the DEDAM concentration at the RCM cycle start in the semi-continuous reactor, for instance. Nevertheless it remained similar when increasing the concentration, that could match more or less to the encountered case during continuous extraction of permeate with a low conversion rate. On the other hand, the retention significantly decreased with the concentration. This last situation could be that encountered during the final diafiltration step if part of the DEDAM was not transformed in c-DEDAM at this step of the overall cascade.

At 40 bar, in cross-flow mode, the c-DEDAM retention was constant at $75 \%$ in the overall concentration range of this study. Moreover, c-DEDAM was proved to be an inhibitor of the RCM reaction [39]. This is a favorable argument to achieve the reaction with a continuous extraction of permeate. Finally, due to the low transmission and regardless of the membrane reactor mode, either semi-continuous or continuous, diafiltration steps were obviously needed to recover the maximum product amount at the RCM end.

\subsection{OSN membrane reactor running in semi-continuous mode}

Since the pre-catalyst retention was the highest at 40 bar, this TMP was chosen for the membrane reactor. Two initial concentrations of the pre-catalyst were tested with the same DEDAM initial load in order to vary the substrate to pre-catalyst initial ratio.

\subsubsection{Grubbs-Hoveyda II pre-catalyst at $10^{-3}{\mathrm{~mol} . \mathrm{L}^{-1}}^{-1}$}

\section{Cascade of "RCM cycle + OSN step"}

RCM was first achieved with the Grubbs-Hoveyda II pre-catalyst at an initial concentration of $1.00 \times 10^{-3} \mathrm{~mol} . \mathrm{L}^{-1}$. The substrate (DEDAM) concentration was chosen accordingly, in order to reach a classical substrate to pre-catalyst ratio close to 100 .

The DEDAM first load was $0.032 \mathrm{~mol}$ corresponding to $0.106 \mathrm{~mol} . \mathrm{L}^{-1}$. Figure 10 shows that DEDAM conversion reached a maximum value (Tau $=97 \%)$ in less than 15 min during RCM cycle 1 . Nevertheless, the 1 bar TMP was maintained during 35 min before increasing TMP to 40 bar. In such conditions, the OSN step 1 started on a media of a stable composition.

Figure 11 shows the significant permeate flux decrease with the VRR increase. The precatalyst retention remained similar to that reached when OSN was performed on a single precatalyst solution. About $44 \%$ of the initial amount of the pre-catalyst was still present in the membrane reactor at the end of RCM cycle 1 (UV-Vis). Thus, $56 \%$ of pre-catalyst had been activated during RCM cycle 1 , but this amount was of course deactivated at the end of the cycle due to the lack of DEDAM in the reaction medium. The deactivated form(s) was (were) not identified. It could be drawn that the decrease of the Grubbs-Hoveyda II pre-catalyst 
concentration was mainly due to the RCM reaction itself (and not to a significant loose in permeate during OSN). This important decrease might of course have a significant impact on the following RCM cycle.

About $3 \%$ of the initial amount of DEDAM was always present at the RCM cycle end $(\approx 3 \mathrm{x}$ $10^{-3}$ mol. $\left.\mathrm{L}^{-1}\right)$. Its retention was $80 \%$ meaning that a very little amount of DEDAM crossed the membrane $\left(\approx 6 \times 10^{-4} \mathrm{~mol} \cdot \mathrm{L}^{-1}\right)$, consequently slightly decreasing the purity of c-DEDAM in permeate.

c-DEDAM $\left(\approx 103 \times 10^{-3}\right.$ mol.L'1) was the main constituent of the mixture to be filtered. Its retention was $75 \%$, consequently only a small proportion of the product was recovered in the $200 \mathrm{~mL}$ permeate at $\mathrm{VRR}_{\text {final }} \approx 3$.

Then the second RCM cycle was engaged immediately followed by OSN step 2 and so on.

Figure 10 highlights the conversion rate $\mathrm{Tau}_{\mathrm{t}}$ during the consecutives RCM reactions whereas Table 2 shows the decrease of the maximum conversion rate (Tau) from one RCM cycle to the other.

Figure 11 evidences the progressive decrease of the permeate flux from one OSN step to the following one. Finally, after a cascade of four "RCM cycle + OSN step", $\mathrm{J}_{\mathrm{OSN}} / \mathrm{J}_{0}$ was close to 0.35. A long rinsing step with pure toluene allowed recovering the initial flux of the membrane.

What can be the origin of the drop of the DEDAM conversion ratio from one RCM cycle to the other?

First is the decrease of the catalyst concentration from one RCM cycle to another.

One explanation could be the leaching of activated Ru-containing species during the OSN step, but UV-Vis spectra of permeates revealed no absorbance that could be attributed to such $\mathrm{Ru}$ species. Nevertheless, permeates obtained from the first two "RCM cycle + OSN step" cascades were joined together in a single sample for reactions performed with pre-catalysts 1 , and similarly for reactions performed with pre-catalyst 4. After solvent evaporation the two fractions of c-DEDAM contained in both permeates were submitted to ICP measurements and revealed $\mathrm{Ru}$ contents ranging from 14 to $20 \mathrm{ppm}$ in the dry matter, respectively.

Another explanation could be the natural deactivation of the pre-catalyst. Figure 12 shows the evolution of the UV-Vis spectra of the retentates during the overall cascade of "RCM cycle + OSN step". It is commonly accepted that a certain amount of the pre-catalyst needs first to be converted into its active catalytic form. Since this active form degrades over time and especially in absence of any substrate to be transformed, its concentration is null at the end of each RCM cycle. Thus, for the following RCM cycle, the remaining pre-catalyst must be partly converted to allow the new RCM reaction.

Second is the increase of the substrate to pre-catalyst ratio from one RCM cycle start to the following one. This is due to both the pre-catalyst decrease and the accumulation of unreacted DEDAM in the reactor because of its high retention by the membrane, from one cycle to the other (Table 2). It can be underlined that when only $0.25 \times 10^{-3} \mathrm{~mol}^{-\mathrm{L}^{-1}}$ pre-catalyst remained in the starting RCM medium (RCM cycle 4, Table 2), corresponding to a substrate to pre-catalyst ratio of 660 the conversion was null, even after a 35 min reaction time. 
In order to evaluate the efficiency of the pre-catalyst, the turn over number is classically used. As the pre-catalyst concentration decreased over the RCM cycles, only an apparent TON value could be calculated (equation 3) that reached 249 at the end of RCM cycle 3 (Table 2). On a more scientific point of view, to better underline the pre-catalyst real activity a corrected value $\left(\mathrm{TON}_{\text {corrected }}\right)$ could be estimated from the effective amount of pre-catalyst involved in each cycle (equation 4$)$.

$$
\mathrm{TON}_{\text {corrected, cycle i }}=\mathrm{DEDAM}_{\text {transformed, cycle i }} / \text { pre-catalyst }{ }_{\text {activated, cycle i }}
$$

\section{With:}

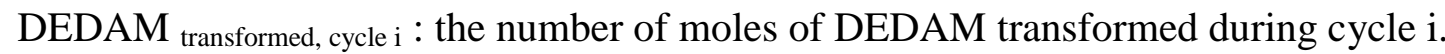

pre-catalyst ${ }_{\text {activated, cycle } \mathrm{i}}$ : the number of moles of pre-catalyst that was activated during cycle i.

To have an overview of the cascade a cumulative value, further called $\mathrm{TON}_{\text {corrected }}$ was calculated as the sum of all the $\mathrm{TON}_{\text {corrected, cycle } \mathrm{i}}$ over the consecutives RCM cycles. TON $_{\text {corrected }}$ reached 1940 at the end of RCM cycle 3 (Table 2).

Third is the increase of c-DEDAM concentration at start of each RCM reaction (Table 2) because of its quite high retention by the membrane and the low final reachable VRR obtained with this equipment. Remember that the product is an inhibitor of the RCM reaction.

\section{What about the deactivated catalyst?}

As already said, the evolution of the pre-catalyst was followed by the UV-Vis spectra of the RCM medium (Figure 12). To better understand the origin of the observed changes, the UVVis spectrum of the free isopropoxystyrene ligand was also recorded. It exhibited a maximum absorbance located at $300 \mathrm{~nm}$ and no absorbance in the 330-450 $\mathrm{nm}$ range (Figure 13a).

Since it was checked that neither DEDAM nor c-DEDAM absorbed UV light in the 290-450 $\mathrm{nm}$ range, spectra of Figure 12 highlight the decomposition of the pre-catalyst as seen from the intensity decrease at $377 \mathrm{~nm}$ and the release of the free isopropoxystyrene ligand as seen from the intensity increase at $300 \mathrm{~nm}$.

Theoretical spectra of mixtures of pure Grubbs-Hoveyda II pre-catalyst and pure free isopropoxystyrene ligand were calculated from the UV-Vis experimental spectra of the two, keeping an overall concentration of $10^{-3} \mathrm{~mol} . \mathrm{L}^{-1}$ (Figure 13a).

Then, experimental spectra of the RCM medium were compared to the theoretical ones (Figure 13b, Figure 13c). Spectra of the RCM medium at the end of RCM cycle 2 and cycle 3 did not exactly match with the theoretical calculations. Moreover, in the 310-340 nm range, a new very large band of absorption appeared, that could only be attributed to entities coming from the pre-catalyst decomposition, that were a priori either active or deactivated forms. Nevertheless, considering that the deactivation step was quick and taking into account the time needed to register each spectrum, then it was more plausible that this new absorption was due to the deactivated entities. The overall retention of "these deactivated entities" was estimated from the absorbance at $330 \mathrm{~nm}$ to be close to $90 \%$. If the assumption concerning the identification of the deactivated form(s) was right, then the amount of $\mathrm{Ru}$ in permeates, issued from both the deactivated form(s) of the pre-catalyst and the pre-catalyst itself, might be quite low. No more comments will be added about theses entities in the following but a 
systematic study will be important to perform in order to take advantage of UV-Vis as an insitu sensor for the mastering of the reactor.

\section{What about the product recovery?}

A better recovery of the product by the OSN step could be obtained if a diafiltration step was added. Diafiltration consists in the addition of a given volume of toluene, which is solvent consuming and finally not fairly good for a sustainable production, except if solvent recycling is included in the overall process (that is probably possible but out of the scope of this study). That is why a systematic diafiltration step was not systematically performed after each "RCM cycle + OSN step". We have chosen to perform two diafiltration steps only on the final medium when no more conversion of the substrate was possible (Figure 5). The added volume of toluene $(200 \mathrm{~mL})$ was more or less arbitrary chosen due to the dead volume of the OSN loop. Of course the diafiltration step could be optimized by controlling the toluene amount and its addition in a continuous mode.

At the end of the cascade of the 3 first RCM cycles, only $32 \%$ of the produced c-DEDAM was recovered in the cumulate permeate (sum of permeate 1 to permeate 3, Figure 5). It can be underlined that because the fourth RCM cycle was un-productive, the OSN step 4 could, in fact, be considered as a first diafiltration step.

After 3 diafiltration steps (OSN step $4+2$ diafiltrations) performed with $3 \times 200 \mathrm{~mL}$ toluene in a semi-continuous mode achieved up to $\mathrm{VRR}_{\text {final }} \approx 3$, only $41 \%$ of the overall c-DEDAM was recovered in the permeate (sum of permeate 1 to permeate 6) that was clearly not sufficient.

Assuming that the c-DEDAM retention remained constant to $75 \%$, it could be drawn that 18 consecutive diafiltration steps should be performed to entirely recover this product by applying the chosen protocol. This appeared as un-realistic and an optimization of the product recovery should be specifically studied since it appeared as a severe limitation for the membrane reactor use. On a practical point of view, we must say that in addition to the cDEDAM high retention, many limitations were due to our equipment and its important dead volume.

Nevertheless, according to this problem, the choice of the membrane and of the filtration conditions that were primarily chosen on the high retention of the pre-catalyst must be questioned. It appeared logically that a compromise must be found between the high retention of the pre-catalyst (and sometimes of the substrate) and the low retention of the product in order to design a sustainable and eco-friendly overall process. (Of course the high retention of the product could appear as non surprising because of the membrane MWCO, but as the retention of the pre-catalyst was quite far from this notion, we were not convinced in the beginning of the study that the DEDAM and c-DEDAM retentions would be so high. Many questions about the transfer mechanism of the pre-catalyst remain open up to now).

\subsubsection{Impact of the pre-catalysts structure at $0.5 \times 10^{-3} \mathrm{~mol}^{-\mathrm{L}^{-1}}$}

An important field of research for chemists involved in organo-metallic synthesis and in homogeneous catalysis is the design of prototype pre-catalysts that must be more and more active. Ideally, according to this strategy they could be let in the final recovered product 
because they would be used in a homeopathic amount in the synthesis media and consequently the remaining metal amount issued from the deactivation would be low (but not null).

According to our own vision, we have tried to find new pre-catalysts that were highly active in the RCM reaction. Moreover these pre-catalysts or their active/deactivated forms might be more retained by the membrane than those issued from the original Grubbs-Hoveyda II precatalyst. The selected prototype pre-catalysts were those shown in Figure 2.

Aiming also at the RCM intensification, the substrate to pre-catalyst initial ratio was increased to 200 (instead of 100 in the previous paragraph). Only the initial concentration of the precatalyst was decreased, down to $0.5 \times 10^{-3} \mathrm{~mol} . \mathrm{L}^{-1}$, whereas each load of DEDAM remained $0.032 \mathrm{~mol}\left(106 \times 10^{-3} \mathrm{~mol} . \mathrm{L}^{-1}\right.$, in $300 \mathrm{~mL}$ toluene $)$. The overall scheme of the process was that shown in Figure 5, except that only 3 RCM cycles were attempted because no conversion of DEDAM was obtained during the RCM cycle 3, regardless of the pre-catalyst. Then a single diafiltration step was performed. Finally it can be considered that 2 diafiltrations were performed when taking into account the un-productive last RCM cycle, as explained above.

Roughly and unfortunately, the modifications of the pre-catalyst did not significantly change the pre-catalyst retention [39, 40]. Nevertheless, the percentage of the activated part varied with the pre-catalyst structure, opening different possibility for the reactor management. Figure 14 summarizes the maximum conversion ratio of DEDAM depending on the RCM cycle for the set of pre-catalysts.

Table 3 compares results obtained with the different pre-catalysts, in which the overall yield of the DEDAM transformation was calculated according to equation 5.

$$
\text { Yield }=\text { DEDAM }_{\text {involved }} / \text { DEDAM }_{\text {transformed }}
$$

With:

DEDAM involved: the mole number of DEDAM engaged in the successive RCM reactions. Because Tau was null at (i) RCM cycle 4 with Grubbs-Hoveyda II pre-catalyst initially at $10^{-3}$ mol.L $\mathrm{L}^{-1}$, (ii) RCM cycle 3 with pre-catalysts $1,2,4$ at $0.5 \times 10^{-3}$ mol. $\mathrm{L}^{-1}$ and (iii) RCM cycle 2 with pre-catalyst 3 at $0.5 \times 10^{-3}$ mol.L $\mathrm{L}^{-1}$, the overall amount of involved DEDAM was taken to be $3 \times 0.032 \mathrm{~mol}, 2 \times 0.032 \mathrm{~mol}$ and $1 \times 0.032 \mathrm{~mol}$, respectively.

DEDAM transformed was the amount of DEDAM transformed in c-DEDAM (single product of the $\mathrm{RCM}$ reaction)

The best yield was obtained with the pre-catalyst 3 that unfortunately was also the less productive one as only one RCM cycle could be achieved.

The percentage of produced c-DEDAM recovered after the cascade of "RCM cycle + OSN step" was close to $25 \%$, for pre-catalysts $1,2,4$ initially at $0.5 \times 10^{-3} \mathrm{~mol} . \mathrm{L}^{-1}$. Finally after the diafiltration steps, the best recovery of c-DEDAM was obtained for the experiment performed with pre-catalyst 2.

The purity of c-DEDAM in the overall permeate was estimated from Equation 2, but in order to take into account the absence of DEDAM transformation during RCM cycle 2, 3 or 4 depending on the pre-catalyst used, only the DEDAM involved in productive RCM cycles 
was taken into account (meaning that $0.032 \mathrm{~mol}$ of DEDAM added during the last RCM cycle but let un-reacted were deduced from the overall remaining DEDAM in the final mixture).

The recovery of the product did not exceed $57 \%$, which mean that this step should be optimized as discussed above. And finally the c-DEDAM purity varied from 77 to $93 \%$.

The main drawn conclusion was that problems evoked with the Grubbs-Hoveyda II precatalyst remained the same regardless of the pre-catalyst. Nevertheless, some advantages could be expected from the design of the pre-catalyst when dealing with the overall yield and the purity of the product.

\subsection{OSN membrane reactor running in continuous mode}

In the previous experiments, when the substrate addition was stopped, the active catalyst remaining in the medium was immediately deactivated. On the other hand, the RCM cycle was only stopped when the maximum DEDAM conversion was reached.

Could it be possible to enhance the active catalyst lifetime by a better control of the substrate addition? To contribute to answer this question, preliminary experiments of RCM achieved in a membrane reactor running in a continuous mode were performed.

In this part, the amount of the Grubbs-Hoveyda II pre-catalyst was the same as what was used in the second set of experiments $\left(1.6 \times 10^{-4} \mathrm{~mol}\right)$ achieved in the semi-continuous mode, but due to the design of the reactor, the initial concentration of pre-catalyst in the loop was, in fact, higher than in the semi-continuous mode. The engaged DEDAM amount was 0.032 mol that corresponded to one RCM cycle in the previous set of experiments (a more important load was not possible due to the observed DEDAM conversion, see below). As detailed in the experimental part, two HPLC pumps were used to feed the OSN loop (Figure 6). Pump 2 provided the DEDAM solution at a constant flow rate and in order to maintain the target pressure, the flux reduction due to the fouling was compensated by the reduction of the flow rate of pump 1 delivering pure toluene. It is noteworthy that this continuous toluene addition can be considered as a continuous diafiltration.

Two conditions were tested during the RCM. The first one was TMP $=40$ bar for which retentions of both the pre-catalyst and the c-DEDAM were quite high $(99.5 \%$ and $75 \%$, respectively). For sake of a better transfer of c-DEDAM toward permeate side, the second experiment was achieved at TMP $=10$ bar for which the c-DEDAM retention was expected to be lower than at 40 bar.

\subsubsection{RCM at 40 bar}

First, the overall amount of the Grubbs-Hoveyda II pre-catalyst was injected in the OSN loop where it remained confined (Figure 6). Then the introduction of DEDAM in the loop of OSN was achieved at $0.10 \mathrm{~mL} \cdot \mathrm{min}^{-1}$, which corresponded to $9.65 \times 10^{-5} \mathrm{~mol} . \mathrm{min}^{-1} .300 \mathrm{~min}$ were needed to inject the overall amount of DEDAM.

Figure 15 shows the continuous decrease of the permeate flux during $200 \mathrm{~min}$. Consequently the residence time of the substrate in the OSN loop varied during the RCM and progressively increased from 35 to $50 \mathrm{~min}$. 
Simultaneously, the c-DEDAM concentration continuously increased in permeate, up to a maximum value reached at $200 \mathrm{~min}$. The shape of this curve is in good accordance with what was observed by Schoeps et al. [30]. The authors attributed the reduction of the conversion to the increasing ethene concentration in the reaction volume that should induce a slow down of the catalytic activity. In the beginning, no DEDAM was transmitted in permeate, but after 150 min its concentration significantly increased. This behavior was in good accordance with a lower catalytic activity in the OSN loop. At $300 \mathrm{~min}$ the overall c-DEDAM recovered in permeate was $7.7 \times 10^{-3} \mathrm{~mol}$ whereas simultaneously $1.4 \times 10^{-3} \mathrm{~mol}$ of DEDAM were also transmitted.

To enhance the c-DEDAM transmission, TMP was first decreased to 20 bar during 150 min. As expected, thanks to the decrease of its retention with the TMP, the c-DEDAM concentration increased in permeate. The concomitant increase of DEDAM clearly revealed the important loss of the catalytic activity. At $450 \mathrm{~min}$ the overall recovery of c-DEDAM in permeate was $10.8 \times 10^{-3}$ mol whereas that of DEDAM was $3.9 \times 10^{-3} \mathrm{~mol}$.

Aiming at enhancing the c-DEDAM recovery a second decrease of the TMP was then applied, down to 10 bar during $55 \mathrm{~min}$. At this TMP the DEDAM retention was $61 \%$ whereas the c-DEDAM retention was only $31 \%$ (instead of $75 \%$ at 40 bar). After 500 min, the overall recovery of c-DEDAM in permeate was $11.5 \times 10^{-3}$ mol and that of DEDAM was $4.8 \times 10^{-3}$ mol.

After the recovery of the remaining retentate and its analysis, the overall amount of cDEDAM obtained during RCM was shown to be $15.2 \times 10^{-3}$ mol; this mean that the conversion rate of DEDAM was quite poor with this system (Tau $=48 \%)$.

The initial membrane flux was easily recovered by the toluene rinsing step; this means that the flux reduction was mainly due to the (reversible) concentration polarization. Consequently, it was quite surprising to observe a black deposit on the demounted membrane that underlined the presence of an irreversible fouling, which was not evidenced by the flux measurements (Figure 16). SEM-EDX analyses evidenced the presence of Ru element in the deposit, which can only correspond to solid un-activated species of the catalyst that were retained by the membrane.

\subsubsection{RCM at 10 bar}

This experiment was performed in a quite similar way to the previous one. First, the overall amount of pre-catalyst Grubbs-Hoveyda II was injected in the OSN loop at 40 bar where it remained confined (Figure 6). Then the pressure in the loop was reduced to $10 \mathrm{bar}$ and the introduction of DEDAM was achieved at $0.10 \mathrm{~mL} \cdot \mathrm{min}^{-1}$, which corresponded to $9.65 \times 10^{-5}$ mol.min ${ }^{-1} .300$ min were needed to inject the overall amount of DEDAM. Due to the applied pressure, the amount of pure toluene introduced with the pump 1 was relatively limited.

Figure 17 shows the permeate flux decrease with time. Moreover, the $\mathrm{J}_{\mathrm{OSN}} / \mathrm{J}_{0}$ variation at 10 bar was close to that at 40 bar. The permeate flux during RCM was 4 times lower at 10 bar than at 40 bar, consequently the residence time of the substrate in the OSN loop increased by the same factor. During the reaction, the retention of DEDAM and c-DEDAM were $71 \%$ and $42 \%$, respectively. 
At 300 min the c-DEDAM amount recovered in permeate was $8.6 \times 10^{-3}$ mol whereas the leakage of DEDAM was $1.2 \times 10^{-3}$ mol. The filtration was maintained during 30 min with injection of pure toluene (continuous diafiltration) and finally $9.6 \times 10^{-3} \mathrm{~mol}$ of c-DEDAM and $1.4 \times 10^{-3} \mathrm{~mol}$ of DEDAM were recovered in permeate.

At the end, an additional analysis of the retentate allowed to evidence that $27.5 \times 10^{-3} \mathrm{~mol}$ of c-DEDAM have been formed during the overall RCM. Thus the DEDAM conversion was quite high $(\mathrm{Tau}=86 \%)$.

\subsection{Comparison of the two membrane reactors}

Table 4 compares the results obtained with the continuous and the semi-continuous membrane reactors, especially when $1.6 \times 10^{-4}$ mol of the Grubbs-Hoveyda II pre-catalyst were engaged together with $0.032 \mathrm{~mol}$ of DEDAM.

A similar TON was obtained with both the semi-continuous membrane reactor at 10 bar and the continuous membrane reactor at 40 bar, but the time to reach these values was significantly different. With the continuous reactor, the RCM duration was two times that in the semi-continuous reactor, but it must be underlined that the continuous membrane reactor was not optimized. Nevertheless the c-DEDAM amount recovered before the diafiltration steps was higher with the continuous reactor, another advantage of which was the lower amount of solvent used.

\section{Conclusion}

Industrial applications of olefin metathesis using homogeneous (soluble) catalysts are often limited due to the high cost of these catalysts, especially if they are used in a single reaction without any possible reuse.

This paper focused on the integration of organic solvent nanofiltration to enhance the catalyst productivity. The main chosen pre-catalyst was a commercial one, namely Grubbs-Hoveyda II. First, on a catalytic point of view our results were in good agreement with those published by Vorfalt et al. [32] highlighting the natural and irreversible degradation of the pre-catalyst in contact with a substrate (here DEDAM). These results underlined that no recycling of the pre-catalyst would be possible contrary to what was initially believed from the release/return boomerang mechanism.

For the first time according to the best of our knowledge, taking into account this new information, together with the fact that the active catalyst form was not long time stable in absence of any substrate, we have compared the performances of two different membrane reactors, expecting at enhancing the catalyst productivity.

It was shown for a model RCM reaction that the two types of membrane reactors could reach similar performances. A main difference would be the process time (more favorable for the semi-continuous mode) and the solvent volume to be used (more favorable for the continuous mode). 
An important limitation of the two membrane reactors, for this model reaction, was the recovery of the final product. In this study, as often proposed in the literature, the membrane was first selected on a single criterion: "to have a high retention of the pre-catalyst". It should be probably better adapted to look for a compromise between the high retention of the precatalyst and the high transmission of the product in order to limit the following diafiltration steps, which were both time and solvent consuming.

Of course this compromise depends on the metathesis substrate and products and the optimization must be done on each specific medium. To transpose these first results, olefin metathesis achieved on a substrate of a renewable origin performed in OSN membrane reactors is currently in progress in our team.

\section{Acknowledgements}

This study was supported by the French National Agency of Research (ANR) through the ANR-09-CP2D-11-01 (NanoRemCat2) program. The authors acknowledge Dr Cédric Fischmeister, UMR-CNRS Institut des Sciences Chimiques de Rennes for helpful discussions on RCM. Francis Gouttefangeas, from CMEBA of University Rennes 1 (http://www.cmeba.univ-rennes1.fr) has performed SEM-EDX analysis of membranes.

\section{References}

[1] A.I. Shafer, A.G. Fane, T.D. Waite, Nanofiltration, Principles and Applications. (2006), Elsevier, Oxford, UK.

[2] L.S. White, Transport properties of a polyimide solvent resistant nanofiltration membrane, J. Membrane Sci., 205 (2002) 191-202.

[3] R.M. Gould, L.S. White, C.R. Wildemuth, Membrane Separation in Solvent Lube Dewaxing, Env. Progress, 20 (2001) 12-16.

[4] S. Darvishmanesh, L. Firoozpour, J. Vanneste, P. Luis, J. Degreve, B. Van der Bruggen, Performance of solvent resistant nanofiltration membranes for purification of residual solvent in the pharmaceutical industry: experiments and simulation, Green Chem., 13 (2011) 34763483 .

[5] G. Székely, J. Bandarra, W. Heggie, B. Sellergen, F.C. Ferreira, Organic solvent nanofiltration: A platform for removal of genotoxins from active pharmaceutical ingredients, J. Membrane Sci., 381 (2011) 21-33.

[6] I. Sereewatthanawut, F.W. Lim, Y.S. Bhole, D. Ormerod, A. Horvath, A.T. Boam, A.G. Livingston, Demonstration of Molecular purification in Polar Aprotic solvents by Organic Solvent Nanofiltration, Org. Process Res. Dev., 14 (2010) 600-611.

[7] D. Ormerod, Applications of organic solvent nanofiltration in the process development of active pharmaceutical ingredients, Proceeding of innovation for sustainable production i-SUP, April 22-25, (2008), Bruges (Belgium), 1-25. 
[8] J. Geens, B.D. Witte, B. Van der Bruggen, Removal of API's (Active Pharmaceutical Ingredients) from organic solvents by nanofiltration, Sep. Sci. Technol., 42 (2007) 2435-2449.

[9] http://www.Kochmembrane.com/applications/pharm.html.

[10] H. Yacubowicz, J. Yacubowicz, Nanofiltration: properties and uses, Filtration Separation, 42 (2005) 16-21.

[11] http://www.membrane-extraction-technology.com/.

[12] P. Vandezande, L.E.M. Gevers, I.F.J. Vankelecom, Solvent resistant nanofiltration: separating on a molecular level, Chem. Soc. Rev., 37 (2008) 365-405.

[13] J.T. Scarpello, D. Nair, L.M. Freitas dos Santos, L.S. White, A.G. Livingston, The separation of homogeneous organometallic catalysts using solvent resistant nanofiltration, $\mathrm{J}$. Membrane Sci., 203 (2002) 71-85.

[14] D. Nair, J.T. Scarpello, I.F.J. Vankelecom, L.M. Freitas dos Santos, L.S. White, R.J. Kloetzing, T. Welton, A.G. Livingston, Increased catalytic productivity for nanofiltrationcoupled Heck reactions using highly stable catalysts systems, Green Chem., 4 (2002) 319-324.

[15] H.P. Dijkstra, G.P.M. van Klink, G. van Koten, The use of ultra- and nanofiltration techniques in homogeneous catalyst recycling, Acc. Chem. Res., 35 (2002) 798-810.

[16] D. Nair, S.S. Luthra, J.T. Scarpello, I.F.J. Vankelecom, L.S. White,A.G. Livingston, A.T. Boam, Homogeneous catalyst separation and re-use through nanofiltration of organic solvents, Desalination, 147 (2002) 301-306.

[17] K. de Smet, S. Aerts, E. Ceulemans, I.F.J. Vankelecom, P.A. Jacobs, Nanofiltrationcoupled catalysis to combine the advantages of homogeneous and heterogeneous catalysis, Chem. Commun., (2001) 597-598.

[18] D. Nair, J.T. Scarpello, L.S. White, L.M. Freitas dos Santos, I.F.J. Vankelecom, A.G. Livingston, semi continuous nanofiltration Heck reactions as a new approach to improve productivity of homogeneous catalyst, Tetrahedron Lett., 4 (2001) 8219-8222.

[19] R.H. Grubbs (Ed), Handbook of Metathesis. (2003), Volume 1-3 Wiley-VCH, Weinheim, Germany.

[20] B. Cornils, W.A. Hermann, I.T. Horvath, W. Leitner, S. Mecking, H. Olivier-Bourbigou, D. Vogt (Eds), Multiple homogeneous catalysis. (2005), Wiley-VCH, Weinheim, Germany.

[21] C. Thurier, C. Fischmeister, C. Bruneau, H. Olivier-Bourbigou, P. Dixneuf, Ionic imidazolium containing ruthenium complexes and olefin metathesis in ionic liquid, J. Mol. Catal., 268 (2007) 127-133.

[22] N. Audic, H. Clavier, M. Mauduit J.C. Guillemin, An ionic liquids-supported ruthenium carbene complex : a robust and recyclable catalyst for ring closing olefin metathesis in ionic liquid, J. Am. Chem. Soc., 125 (2003) 9248-9249. 
[23] P. Sledz, M. Mauduit, K. Grela, olefin metathesis in ionic liquids, Chem. Soc. Rev., 37 (2008) 2433-2442.

[24] A. Fürstner, L. Ackermann, K. Beck, H. Hori, D. Koch, K. Langemann, M. Liebl, C. Six, WW. Leitner, Olefin metathesis in supercritical carbon dioxide, J. Am. Chem. Soc., 123 (2001) 9000-9006.

[25] F. Michalek, D. Mädge, J. Rühe, W. Bannwarth, Ring closure metathesis in supercritical carbone dioxide as solvent with use of covalently immobilized ruthenium catalysts, Eur. J. Org. Chem. 2006 (2006) 577-581.

[26] Q. Yao, Y. Zhang, Poly(fluoroalkyle acrylate)-Bound Ruthenium Carbene Complex: A fluorous and recyclable catalyst for ring-closing olefin metathesis, J. Am. Chem. Soc., 126 (2004) 74-75.

[27] A. Kajetanowicz, J. Czaban, G.R. Krishnan, M. Malinska, K. Wozniak, H. Siddique, L.G. Peeva, A.G. Livingston, K. Grela, Batchwise and Continuous Nanofiltration of POSSTagged Grubbs-Hoveyda-Type Olefin Metathesis Catalysts, ChemSusChem, 6 (2013) 182192.

[28] P. van der Gryp, A. Barnard, J.-P. Cronje, D. de Vlieger, S. Marx, H.C.M. Vosloo, Separation of different metathesis Grubbs-type catalysts using organic solvent nanofiltration, J. Membrane Sci., 353 (2010) 70-77.

[29] A. Keraani, M. Rabiller-Baudry, C. Fischmeister, C. Bruneau, Immobilisation of an ionically tagged Hoveyda catalyst on a supported ionic liquid membrane: An innovative approach for metathesis reactions in a catalytic membrane reactor, Catal. Today, 156 (2010) 268-275.

[30] D. Schoeps, K.Buhr, M. Dijkstra, K. Ebert, H. Plenio, Batchwise and Continuous Organophilic Nanofiltration of Grubbs-Type Olefin Metathesis Catalysts, Chem. Eur. J., 15 (2009) 2960-2965.

[31] A. Keraani, T. Renouard, C. Fischmeister, C. Bruneau, M. Rabiller-Baudry, Recovery of Enlarged Olefin Metathesis Catalysts by Nanofiltration in an Eco-Friendly Solvent, ChemSusChem, 1 (2008) 927-933.

[32] T. Vorfalt, K.J. Wannowius, V. Thiel, H. Plenio, How Important Is the Release-Return Mechanism in Olefin Metathesis ?, Chem. Eur. J., 16 (2010) 12312-12315.

[33] T. Ritter, A. Hejl, A. Wenzel, T. Funk, R.H. Grubbs, A Standard System of Characterization for Olefin Metathesis Catalysts, Organometallics 25 (2006) 5740-5745.

[34] S. Shahane, L. Toupet, C. Fischmeister, C. Bruneau, Synthesis and Characterization of Sterically Enlarged Hoveyda-Type Olefin Metathesis Catalysts, Eur. J. Inorg. Chem., (2013) 54-60. 
[35] S. Darvishmanesh, A. Buekenhoudt, J. Degrève, B. Van der Bruggen, General model for prediction of solvent permeation through organic and inorganic solvent resistant nanofiltration membranes, J. Membrane Sci., 334 (2009) 43-49.

[36] R. Valadez-Blanco, A.G. Livingston, Solute molecular transport through polyimide asymmetric organic solvent nanofiltration (OSN) membranes and the effect of membraneformation parameters on mass transfer, J. Membrane Sci., 326 (2009) 332-342.

[37] L.E.M. Gevers, G. Meyen, K. De Smet, P. Van De Velde, F. Du Prez, I.F.J. Vankelecom, P.A. Jacobs, Physico-chemical interpretation of the SRNF transport mechanism for solutes through dense silicone membranes, J. Membrane Sci., 274 (2006) 173-182.

[38] D. Nair, H.-T. Wong, S. Han, I.F.J. Vankelecom, L.S. White, A.G. Livingston, A.T. Boam, Extending Ru-BINAP Catalyst Life and Separating Products from Catalyst Using Membrane Recycling, Org. Process Res. Dev., 13 (2009) 863-869.

[39] G. Nasser, T. Renouard, S. Shahane, C. Fischmeister, C. Bruneau, and M. RabillerBaudry, Interest of the pre-catalyst design for olefin metathesis in a discontinuous nanofiltration membrane reactor, ChemPlusChem, 2013, in press.

[40] M. Rabiller-Baudry, G. Nasser, D. Delaunay, A. Keraani, T. Renouard, D. Roizard, H. Ben Soltane, C.Fischmeister, J.L. Couturier, D. Dhaler, Interests and challenges of organic solvent nanofiltration for sustainable chemistry: the case of homogeneous catalysis by metathesis, Proceeding of the Sustainable Chemistry 2011 Conference - 6-8 July 2011, Antwerp, Belgium, 141-152. 


\section{Figure captions}

Figure 1: Mechanism of olefin metathesis reaction according to the "release/return boomerang mechanism" adapted from [30]. Pre-catalyst (stable form) evolves in an active form and a free isopropoxystyrene ligand

Figure 2: Structures of the commercial Grubbs-Hoveyda II pre-catalyst (pre-catalyst 1) and of the prototype pre-catalysts $2-4$.

Figure 3: Ring closing metathesis reaction (RCM) performed at room temperature pre-catalyst initial concentration was $10^{-3}$ mol. $L^{-1}$ or $0.5 \times 10^{-3}$ mol. $L^{-1}$

DEDAM = substrate, initial concentration was 0.106 mol. $L^{-1}$

c-DEDAM = product (cyclopentene form).

Figure 4: Filtration equipment for cross-flow filtration - (a) principle - (b) picture.

Figure 5: Schematic overview of the semi-continuous mode including four successive "RCM cycle + OSN step" (performed up to VRR $\approx 3$ ) and final diafiltration steps to enhance the c-DEDAM recovery. For this cascade, the initial concentration of Grubbs-Hoveyda II pre-catalyst was $10^{-3}$ mol. $L^{-1}$.

Figure 6: Continuous membrane reactor (a) principle - (b) picture.

Feed tank 1 was filled by pure toluene whereas feed tank 2 was filled by DEDAM in toluene

Figure 7: Retention at $V R R_{\text {final }} \approx 3$ of Grubbs-Hoveyda II pre-catalyst in single solution pre-catalyst initial concentration: $0.50 \pm 0.03 \times 10^{-3}$ mol. $L^{-1}$ in toluene

Starmem 122 membrane, $T=26 \pm 3^{\circ} \mathrm{C}$, cross-flow velocity $v \# 0.1 \mathrm{~m} \cdot \mathrm{s}^{-1}$.

Figure 8: Retention at 40 bar of Grubbs- Hoveyda II pre-catalyst in single solution versus the increase of concentration in the feed obtained by continuous increase of VRR up to VRR final $\approx 3$.

[RO] was the initial concentration in toluene in the feed (4 sets of experiments)

Starmem 122 membrane, $T=26 \pm 3^{\circ} \mathrm{C}, \mathrm{TMP}=40 \mathrm{bar}$, cross-flow velocity $\approx 0.1 \mathrm{~m} . \mathrm{s}^{-1}$.

Figure 9: DEDAM retention in cross-flow mode versus its concentration in the feed

Starmem 122 membrane, $T=26 \pm 3^{\circ} \mathrm{C}, T M P=40$ bar, cross-flow velocity $\approx 0.1 \mathrm{~m} . \mathrm{s}^{-1}$.

Figure 10: Evolution of DEDAM conversion $\left(\mathrm{Tau}_{t}\right)$ versus reaction time of $R C M$ at $26^{\circ} \mathrm{C}$ in toluene according to the RCM cycle. A single load of pre-catalyst at $10^{-3}$ mol. $L^{-1}$ Each load of DEDAM was $0.032 \mathrm{~mol}\left(0.106\right.$ mol. $\left.L^{-1}\right)$. One load of DEDAM per RCM cycle + OSN step up to VRR $\approx 3$ at the end of each RCM cycle.

Figure 11. Evolution of the flux reduction during OSN steps of the semi-continuous membrane reactor. A single load of pre-catalyst at $10^{-3} \mathrm{~mol} . \mathrm{L}^{-1}$.

Each load of DEDAM was $0.032 \mathrm{~mol}\left(0.106\right.$ mol. $\left.\mathrm{L}^{-1}\right)$. One load of DEDAM per RCM cycle + OSN step at 40 bar up to $V R R \approx 3$ at the end of each $R C M$ cycle

Figure 12: Evolution of UV-Vis spectra of retentate during RCM cycles performed with $10^{-3}$ mol. $L^{-1}$ initial concentration of Grubbs-Hoveyda II pre-catalyst. Maximum absorption of the pre-catalyst form was measured at $\lambda_{\max }=377 \mathrm{~nm}$. (each spectrum was first acquired after appropriate dilution by toluene to have a maximum absorbance lower than 1 absorbance unit then the dilution correction was done to be able to directly compare the different media). 
Figure 13: UV-Vis spectra of RCM media, Grubbs-Hoveyda II pre-catalyst, free isopropoxystyrene ligand and theoretical calculated spectra of mixtures of the two previous ones. (a) experimental spectra of Grubbs-Hoveyda II and free isopropoxystyrene ligand, both at $10^{-3} \mathrm{~mol}^{-L^{-1}}$, and theoretical calculated spectra of mixtures of the two previous ones (pre-catalyst/ligand) for an overall concentration of $10^{-3}$ mol. $L^{-1 .}(\boldsymbol{b})$ comparison of spectrum of RCM medium at the end of RCM cycle 2 with selected theoretical spectra; (c) comparison of spectrum of RCM medium at the end of RCM cycle 3 with selected theoretical spectra

Figure 14: Maximum DEDAM conversion ratio (Tau) versus RCM cycle in the semi-continuous membrane reactor. A single load of pre-catalyst at either $10^{-3}$ mol. $L^{-1 .}$ or $0.5 \times 10^{-3}$ mol. $L^{-1}$ Each load of DEDAM was $0.032 \mathrm{~mol}\left(0.106 \mathrm{~mol} . \mathrm{L}^{-1}\right)$. One load of DEDAM per RCM cycle + a final OSN step up to $V R R \approx 3$ at the end of each RCM cycle.

Figure 15: RCM performed in the membrane reactor running in continuous mode

(a) evolution of the concentration of DEDAM and c-DEDAM in the permeate

(b) evolution of the flux decrease

During DEDAM addition a 40 bar TMP was applied then TMP was decreased to enhance the product recovery. The pre-catalyst initial load was $1.6 \times 10^{-4}$ mol. The overall load of DEDAM was $0.032 \mathrm{~mol}$. Conditions: $32 \pm 4{ }^{\circ} \mathrm{C}$, cross flow velocity $\approx 0.1 \mathrm{~m} . \mathrm{s}^{-1}$.

Figure 16: Pictures of (a) the pristine Starmem 122 membrane and (b) the Starmem 122 membrane after the RCM reaction at 40 bar in the continuous membrane reactor

(c) SEM-EDX analyses of the two membranes.

Figure 17: RCM performed in the membrane reactor running in continuous mode at 10 bar

(a) evolution of the concentration of DEDAM and c-DEDAM in the permeate

(b) evolution of the flux decrease

pre-catalyst initial load was $1.6 \times 10^{-4} \mathrm{~mol}$. Overall load of DEDAM was $0.032 \mathrm{~mol}$.

Conditions: $32 \pm 4{ }^{\circ} \mathrm{C}$, cross flow velocity $\approx 0.1 \mathrm{~m} . \mathrm{s}^{-1}$. 


\section{Table captions}

Table 1: Flux reduction ( $\left.L p_{\text {final }} L p_{\text {initial }}\right)$ at $V R R \approx 3$ for OSN of Grubbs-Hoveyda II pre-catalyst in dead-end mode. Pre-catalyst initial concentration was $0.50 \pm 0.0310^{-3}$ mol. $L^{-1}$ in toluene. Starmem 122 membrane, $T=26 \pm 3^{\circ} \mathrm{C}$.

Table 2: Maximum conversion rate of DEDAM (Tau) and concentrations of each identified components in the RCM media at start of RCM cycles during the 4 successive RCM reactions in the

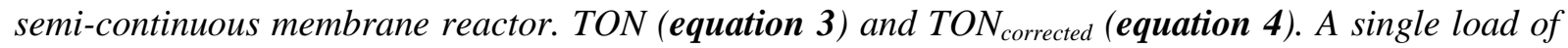
pre-catalyst at $10^{-3}$ mol. $L^{-1 .}$ Each load of DEDAM was $0.032 \mathrm{~mol}\left(0.106 \mathrm{~mol} . \mathrm{L}^{-1}\right)$. One load of DEDAM per RCM cycle + a OSN step at 40 bar up to $V R R \approx 3$ at the end of each RCM cycle

Table 3: Overall results of cascade of RCM cycle + OSN step for the different pre-catalysts

Table 4: Comparison between semi-continuous and continuous mode for a single load of DEDAM (0.032 mol) and of Grubbs-Hoveyda II pre-catalyst 
Table 1: Flux reduction ( $L p_{\text {final }} / L p_{\text {initial }}$ ) at $V R R \approx 3$ for $O S N$ of the Grubbs-Hoveyda II pre-catalyst in dead-end mode. Pre-catalyst initial concentration was $0.50 \pm 0.0310^{-3}$ mol. $L^{-1}$ in toluene. Starmem 122 membrane, $T=26 \pm 3^{\circ} \mathrm{C}$.

\begin{tabular}{|c|c|}
\hline TMP (bar) & $\mathrm{Lp}_{\text {final }} / \mathrm{Lp}_{\text {initial }}$ \\
\hline 40 & 0.06 \\
\hline 30 & 0.18 \\
\hline 20 & 0.24 \\
\hline 10 & 0.35 \\
\hline
\end{tabular}


Table 2: Maximum conversion rate of DEDAM (Tau) and concentrations of each identified components in the RCM media at start of RCM cycles during the 4 successive RCM reactions in the

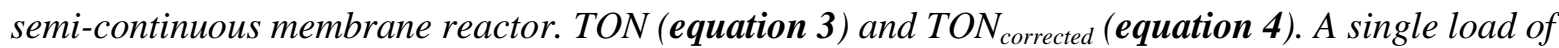
pre-catalyst at $10^{-3}$ mol. $L^{-1 .}$ Each load of DEDAM was $0.032 \mathrm{~mol}\left(0.106 \mathrm{~mol} . \mathrm{L}^{-1}\right)$. One load of DEDAM per RCM cycle + OSN step at 40 bar up to $V R R \approx 3$ at the end of each RCM cycle

\begin{tabular}{|c|c|c|c|c|c|c|}
\hline RCM cycle & $\begin{array}{c}\text { Tau } \\
(\%)\end{array}$ & $\begin{array}{c}\text { pre-catalyst } \\
10^{-3} \mathrm{~mol} . \mathrm{L}^{-1}\end{array}$ & $\begin{array}{c}\text { DEDAM } \\
10^{-3} \text { mol. } L^{-1}\end{array}$ & $\begin{array}{c}\text { c-DEDAM } \\
10^{-3} \text { mol. } L^{-1}\end{array}$ & TON & TON $_{\text {corrected }}$ \\
\hline 1 & 97 & 1.00 & 106 & 0 & 103 & 184 \\
\hline 2 & 85 & 0.44 & 107 & 85 & 194 & 650 \\
\hline 3 & 45 & 0.30 & 123 & 147 & 249 & 1940 \\
\hline 4 & 0 & 0.25 & 165 & 171 & - & - \\
\hline
\end{tabular}


Table 3: Overall results of the cascade of RCM cycle + OSN step for the different pre-catalysts

\begin{tabular}{|c|c|c|c|c|c|c|c|c|}
\hline \multirow{2}{*}{$\begin{array}{c}\text { pre- } \\
\text { catalyst }\end{array}$} & \multirow{2}{*}{$\begin{array}{c}\text { Initial pre- } \\
\text { catalyst } \\
\text { Concentration } \\
\left(10^{-3} \text { mol.L } \mathrm{L}^{-1}\right)\end{array}$} & \multirow{2}{*}{$\begin{array}{l}\text { Overall } \\
\text { added } \\
\text { DEDAM } \\
(1)\end{array}$} & \multirow[t]{2}{*}{ TON } & \multirow{2}{*}{$\begin{array}{l}\text { Yield } \\
(\%)\end{array}$} & \multicolumn{2}{|c|}{$\begin{array}{c}\text { Recovered c-DEDAM } \\
\text { in permeate } \\
(\%)\end{array}$} & \multirow{2}{*}{$\begin{array}{c}\text { Added } \\
\text { toluene for } \\
\text { overall } \\
\text { diafiltration }\end{array}$} & \multirow{2}{*}{$\begin{array}{c}\text { c- } \\
\text { DEDAM } \\
\text { Purity } \\
(\%)\end{array}$} \\
\hline & & & & & $\begin{array}{c}\text { before } \\
\text { diafiltration }\end{array}$ & $\begin{array}{c}\text { after } \\
\text { diafiltration }\end{array}$ & & \\
\hline 1 & 1 & $3 \times 0.032$ & 249 & 78 & $32(2)$ & 41 & $\begin{array}{c}625 \\
\text { (3 diaf) }\end{array}$ & 91 \\
\hline 1 & 0.5 & $2 \times 0.032$ & 230 & 57 & $28(3)$ & 47 & $\begin{array}{c}400 \\
(2 \text { diaf })\end{array}$ & 77 \\
\hline 2 & 0.5 & $2 \times 0.032$ & 309 & 74 & $27(3)$ & 57 & $\begin{array}{c}400 \\
(2 \text { diaf })\end{array}$ & 85 \\
\hline 3 & 0.5 & $1 \times 0.032$ & 179 & 86 & $16(4)$ & 40 & $\begin{array}{c}400 \\
\text { (2 diaf) }\end{array}$ & 93 \\
\hline 4 & 0.5 & $2 \times 0.032$ & 329 & 81 & $25(3)$ & 45 & $\begin{array}{c}400 \\
\text { (2 diaf) }\end{array}$ & 85 \\
\hline
\end{tabular}

(1) only in RCM cycles producing c-DEDAM

(2) 3 "RCM $+O S N "+3$ diafiltrations (DEDAM was added during the first diafiltration step but let un-reacted)

(3) 2 "RCM + OSN" +2 diafiltrations (DEDAM was added during the first diafiltration step but let un-reacted)

(4) 1 "RCM + OSN" +2 diafiltrations (DEDAM was added during the first diafiltration step but let un-reacted) 
Table 4: Comparison between semi-continuous and continuous mode for a single load of DEDAM $(0.032 \mathrm{~mol})$ and of Grubbs-Hoveyda II pre-catalyst

\begin{tabular}{|c|c|c|c|c|c|c|c|}
\hline $\begin{array}{c}\text { Pre- } \\
\text { catalyst } \\
\text { load } \\
(\mathrm{mol})\end{array}$ & $\begin{array}{c}\text { Membrane } \\
\text { reactor }\end{array}$ & $\begin{array}{l}\text { TMP } \\
\text { (bar) }\end{array}$ & $\begin{array}{c}\mathrm{c}- \\
\text { DEDAM } \\
\text { formed } \\
(\mathrm{mol})\end{array}$ & $\begin{array}{c}\text { apparent } \\
\text { TON }\end{array}$ & $\begin{array}{l}\text { c-DEDAM } \\
\text { recovery } \\
\text { before } \\
\text { diafiltration } \\
\quad(\mathrm{mol})\end{array}$ & $\begin{array}{c}\text { Time } \\
\text { before } \\
\text { diafiltration } \\
\text { (h) }\end{array}$ & $\begin{array}{c}\text { Toluene } \\
\text { used before } \\
\text { diafiltration } \\
(\mathrm{mL})\end{array}$ \\
\hline $\begin{array}{c}3.2 \mathrm{x} \\
10^{-4}\end{array}$ & $\begin{array}{c}\text { semi- } \\
\text { continuous }\end{array}$ & 40 & $\begin{array}{c}0.031 \\
0.058(1)\end{array}$ & $\begin{array}{c}97 \\
176\end{array}$ & $\begin{array}{l}5.4 \times 10^{-3} \\
8.9 \times 10^{-3}\end{array}$ & $\begin{array}{l}2(3) \\
4(3)\end{array}$ & $\begin{array}{l}300 \\
500\end{array}$ \\
\hline $\begin{array}{c}1.6 \mathrm{x} \\
10^{-4}\end{array}$ & $\begin{array}{c}\text { semi- } \\
\text { continuous }\end{array}$ & 40 & 0.031 & 194 & $5.1 \times 10^{-3}$ & $2(4)$ & 300 \\
\hline $\begin{array}{c}1.6 \mathrm{x} \\
10^{-4}\end{array}$ & Continuous & 40 & 0.015 & 94 & $\begin{array}{c}7.7 \times 10^{-3} \\
\text { (2) }\end{array}$ & 5 & 57 \\
\hline $\begin{array}{c}1.6 \mathrm{x} \\
10^{-4}\end{array}$ & Continuous & 10 & 0.028 & 172 & $\begin{array}{c}8.6 \times 10^{-3} \\
\text { (2) }\end{array}$ & 5 & 70 \\
\hline
\end{tabular}

(1) 2 loads of DEDAM

(2) at end of DEDAM addition

(3) estimated as diafiltration was performed at end of RCM 4

(4) estimated as diafiltration was performed at end of RCM 3 


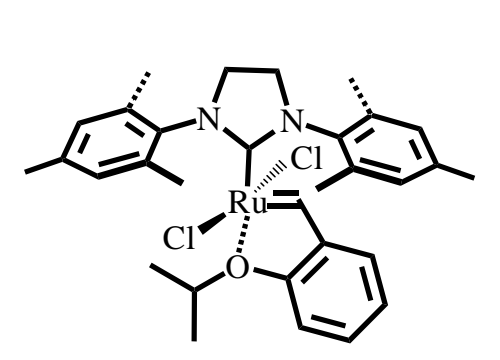

pre-catalyst

(Grubbs-Hoveyda II )
Substrate

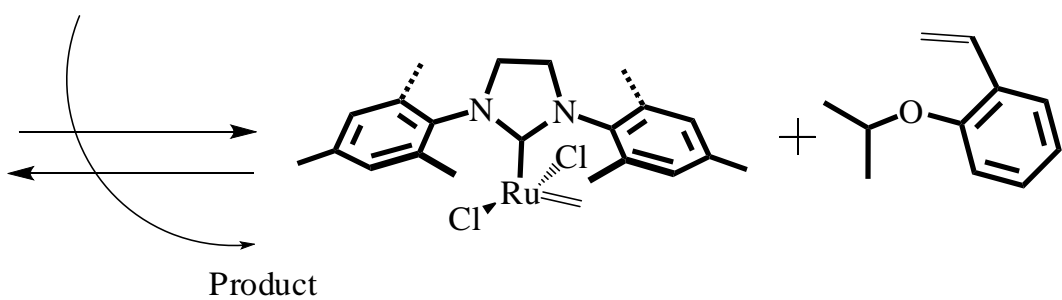

active form

isopropoxystyrene ligand

Figure 1: Mechanism of olefin metathesis reaction according to the "release/return boomerang mechanism" adapted from [30]. Pre-catalyst (stable form) evolves in an active form and a free isopropoxystyrene ligand. 

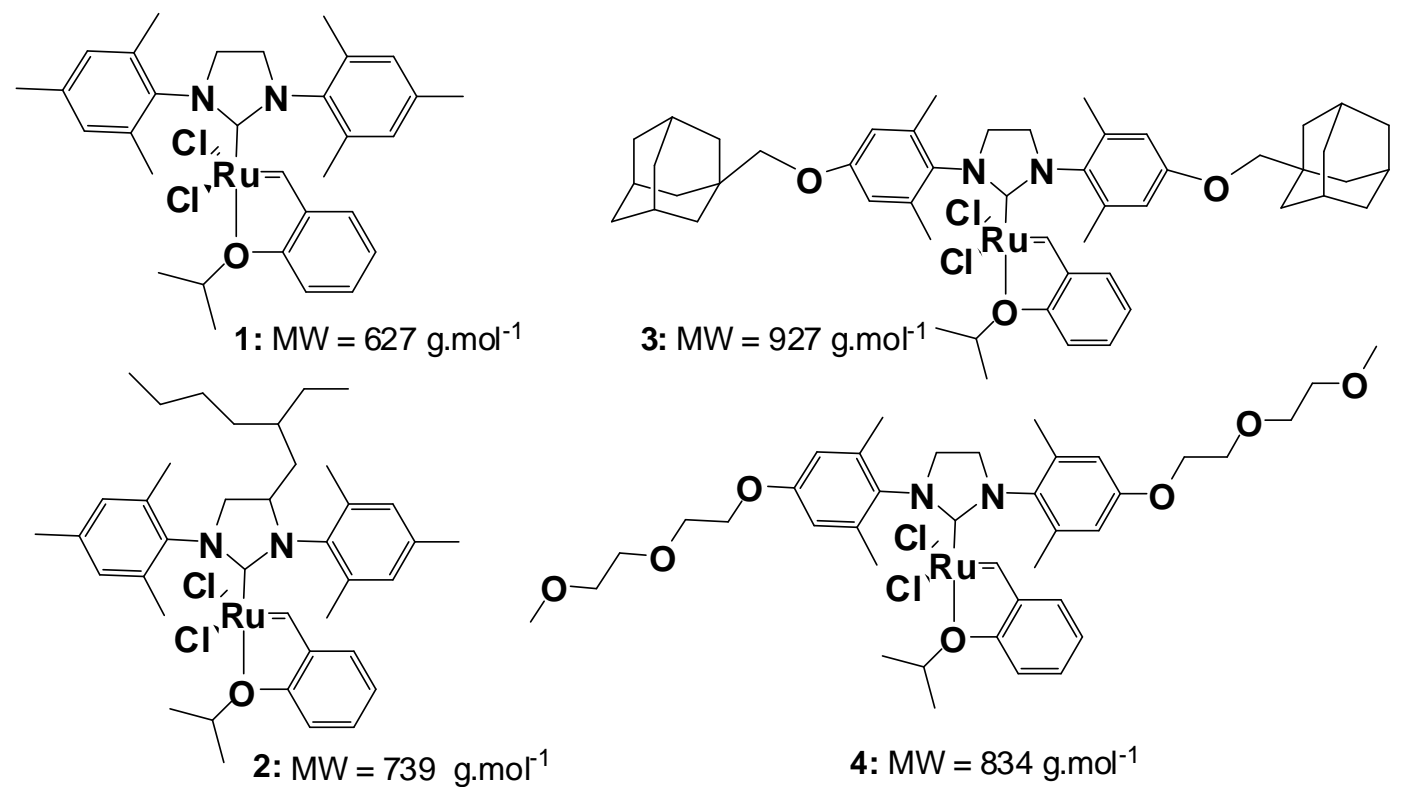

Figure 2: Structures of the commercial Grubbs-Hoveyda II pre-catalyst (pre-catalyst 1) and of the prototype pre-catalysts 2-4. 


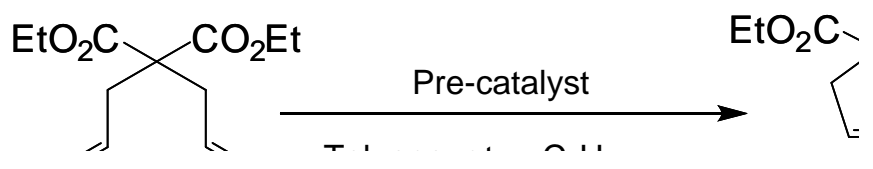

Figure 3: Ring closing metathesis reaction (RCM) performed at room temperature pre-catalyst initial concentration was $10^{-3}$ mol. $L^{-1}$ or $0.5 \times 10^{-3}$ mol. $L^{-1}$ DEDAM = substrate, initial concentration is $0.106 \mathrm{~mol} . \mathrm{L}^{-1}$ c-DEDAM = product (cyclopentene form). 


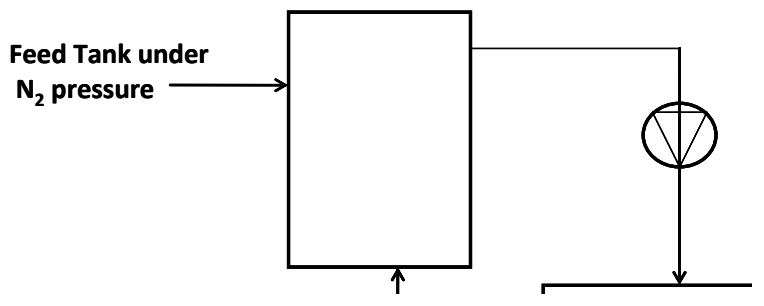

(a)

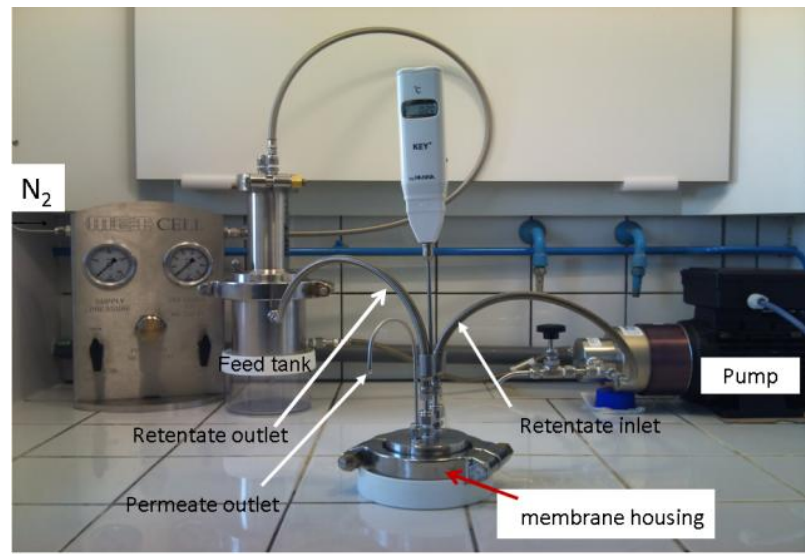

(b)

Figure 4: Filtration equipment for cross-flow filtration (a) principle - (b) picture. 


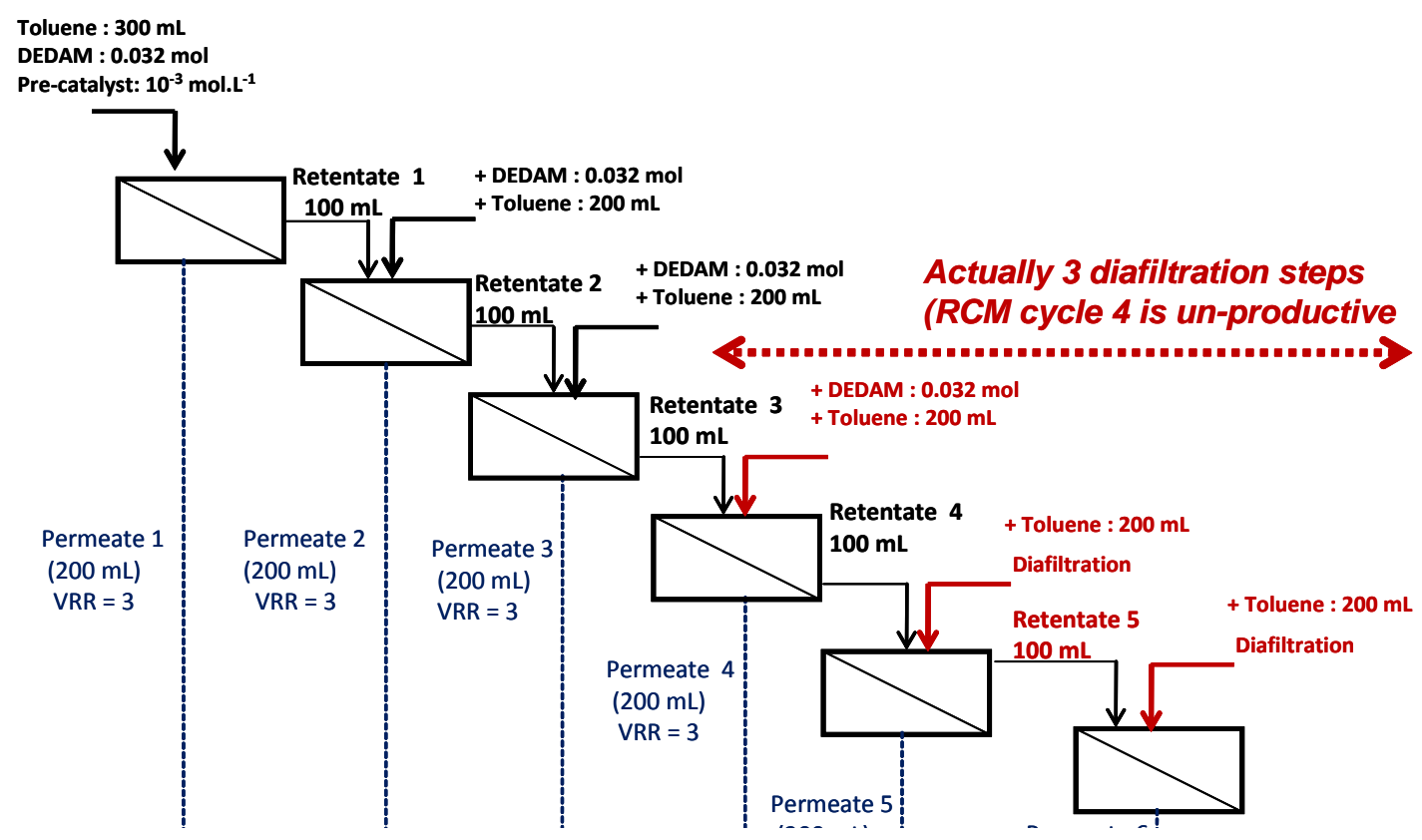

Figure 5: Schematic overview of the semi-continuous mode including four successive "RCM cycle + OSN step" (performed up to VRR $\approx 3$ ) and final diafiltration steps to enhance the c-DEDAM recovery.

For this cascade, the initial concentration of Grubbs-Hoveyda II pre-catalyst was $10^{-3} \mathrm{~mol} . \mathrm{L}^{-1}$. 


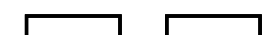

(a)

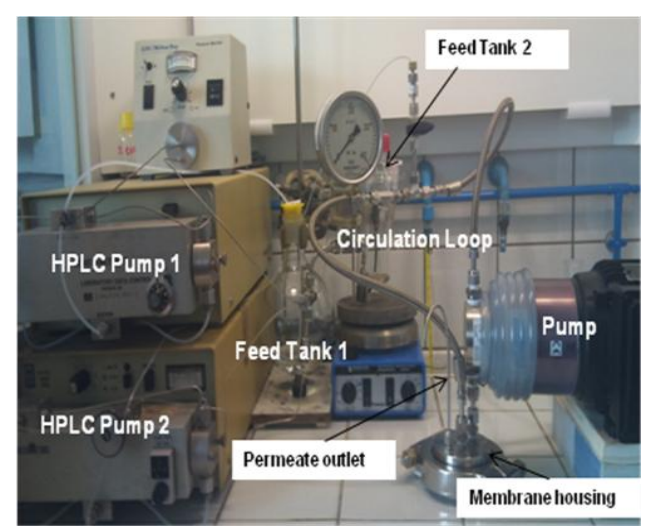

(b)

Figure 6: Continuous membrane reactor (a) principle - (b) picture.

Feed tank 1 was filled by pure toluene whereas feed tank 2 was filled by DEDAM in toluene 


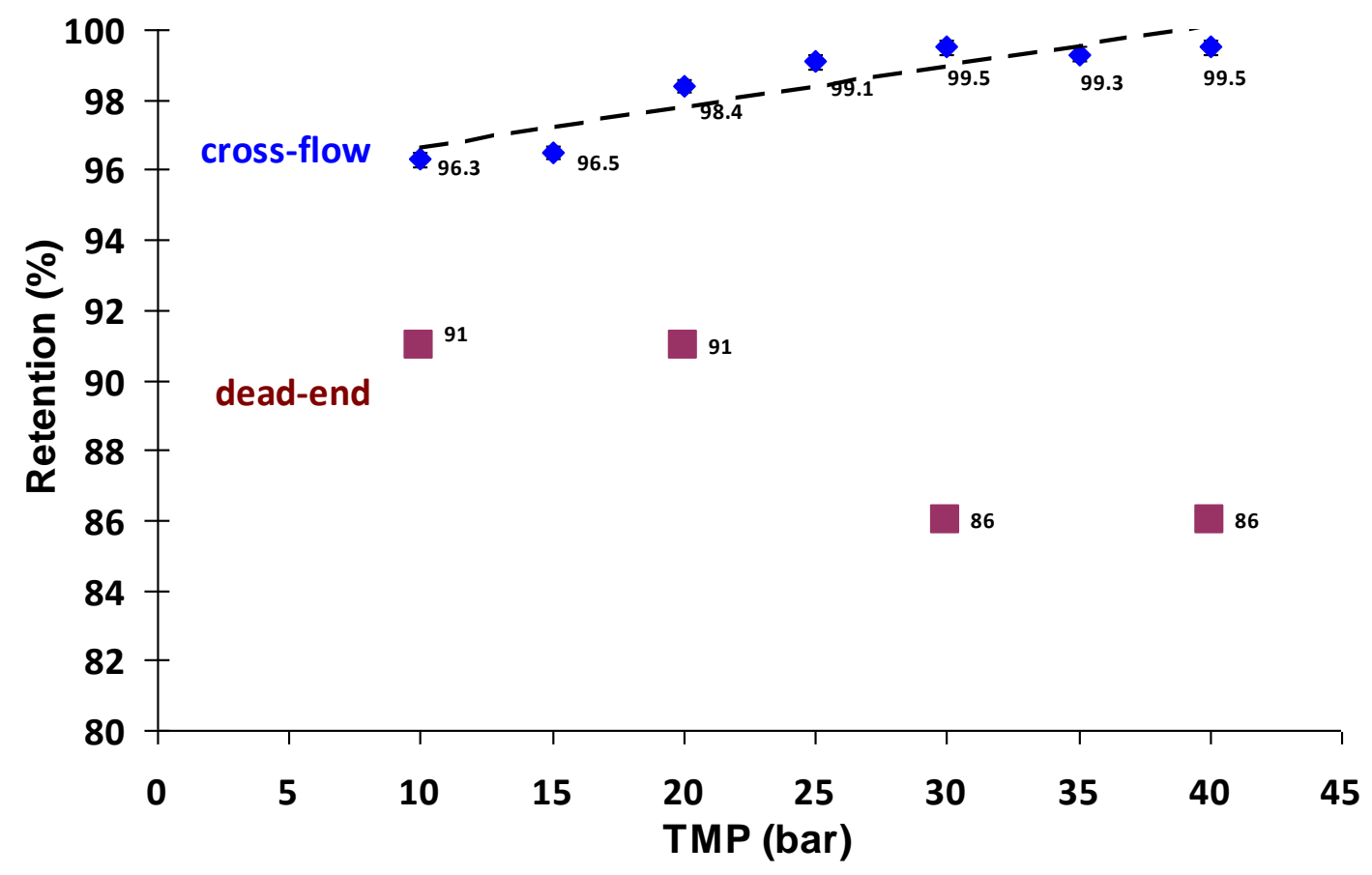

Figure 7: Retention at $V R R_{\text {final }} \approx 3$ of Grubbs-Hoveyda II pre-catalyst in single solution pre-catalyst initial concentration: $0.50 \pm 0.03 \times 10^{-3} \mathrm{~mol} . \mathrm{L}^{-1}$ in toluene Starmem 122 membrane, $T=26 \pm 3^{\circ} \mathrm{C}$, cross-flow velocity $v \# 0.1 \mathrm{~m} . \mathrm{s}^{-1}$. 


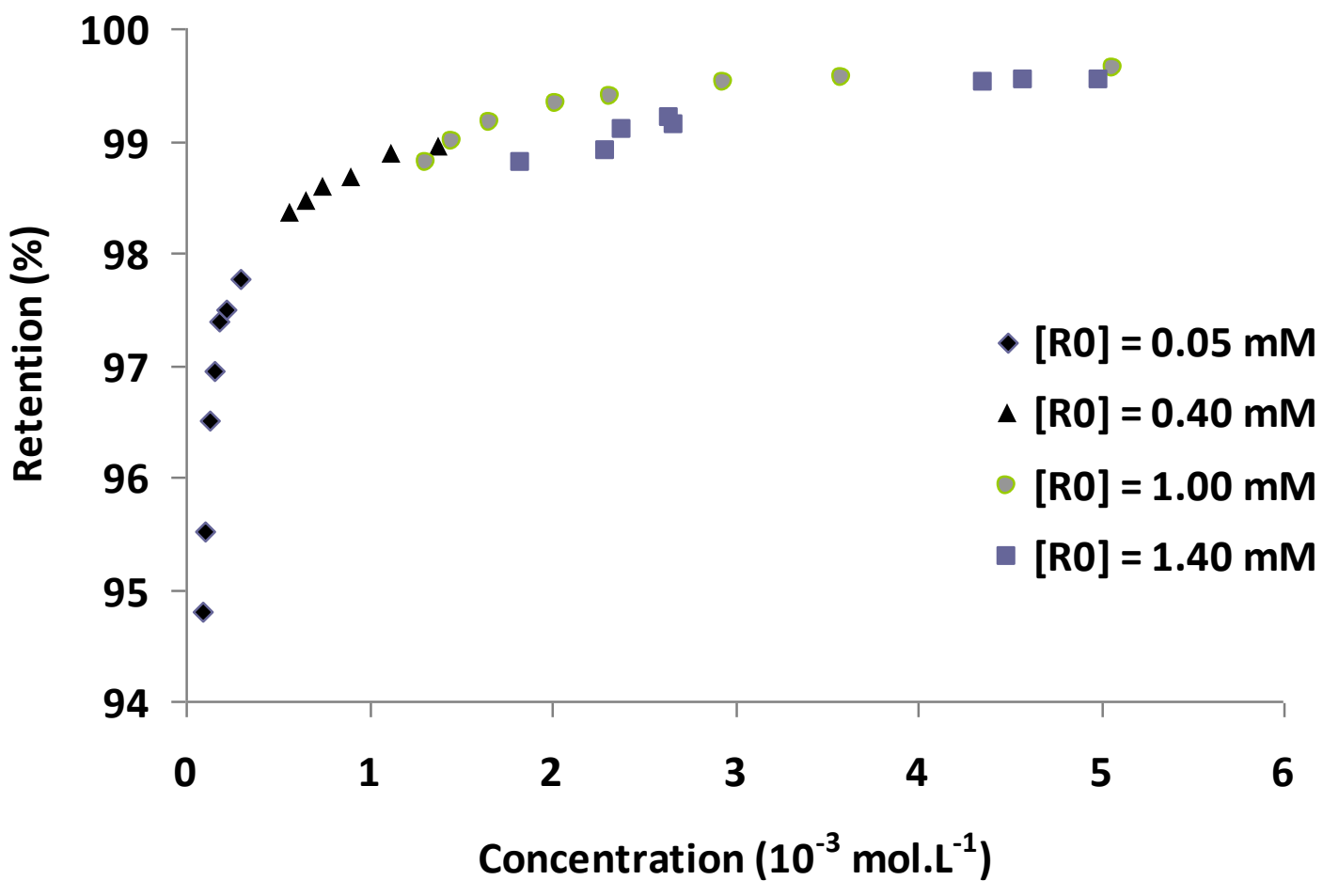

Figure 8: Retention at 40 bar of Grubbs- Hoveyda II pre-catalyst in single solution versus the increase of concentration in the feed obtained by continuous increase of VRR up to VRR fina $\approx 3$.

[RO] was the initial concentration in the feed in toluene (4 sets of experiments) Starmem 122 membrane, $T=26 \pm 3^{\circ} \mathrm{C}, \mathrm{TMP}=40 \mathrm{bar}$, cross-flow velocity $\approx 0.1 \mathrm{~m} . \mathrm{s}^{-1}$. 


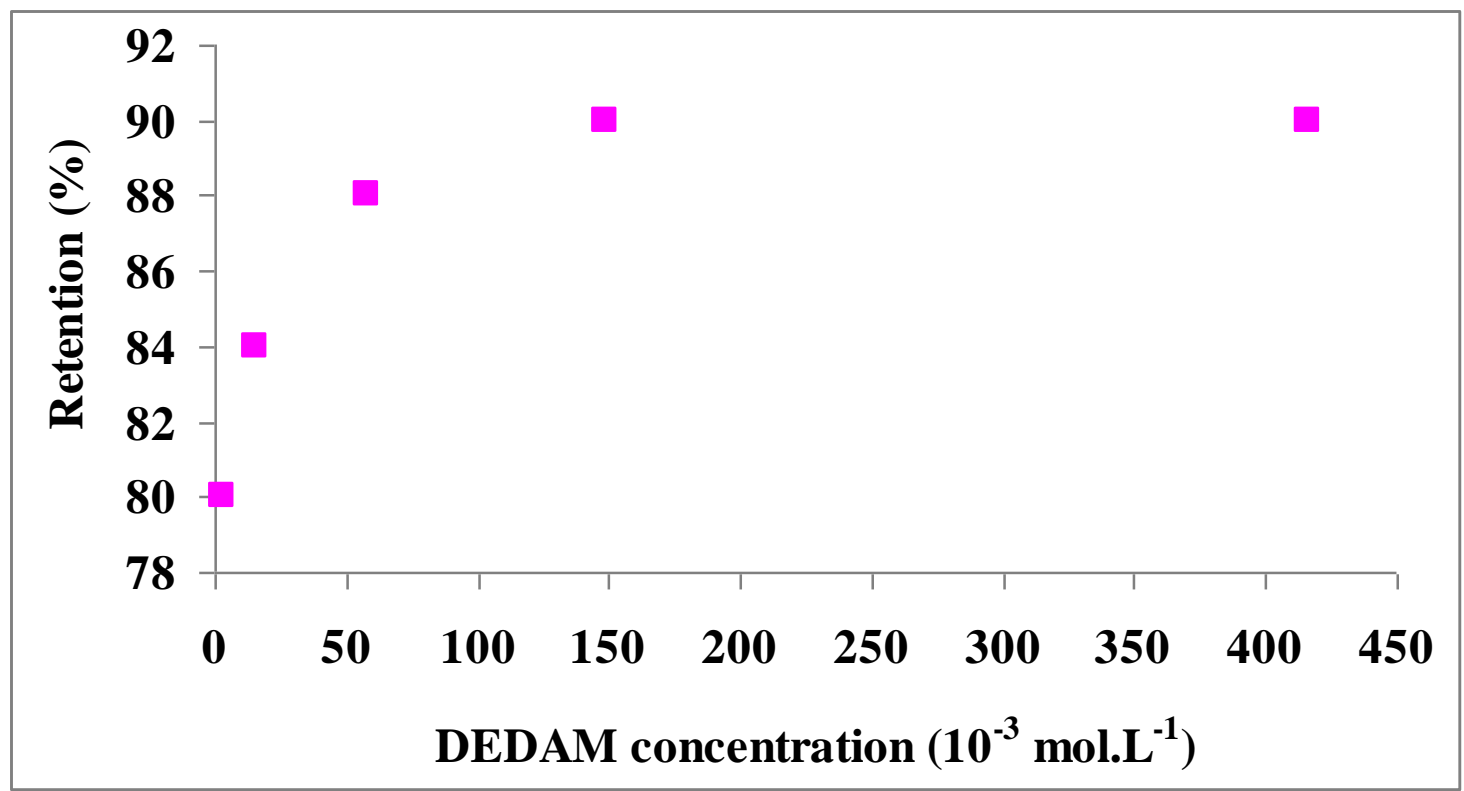

Figure 9: DEDAM retention in cross-flow mode versus its concentration in the feed Starmem 122 membrane, $T=26 \pm 3^{\circ} \mathrm{C}, \mathrm{TMP}=40 \mathrm{bar}$, cross-flow velocity $\approx 0.1 \mathrm{~m} \cdot \mathrm{s}^{-1}$. 


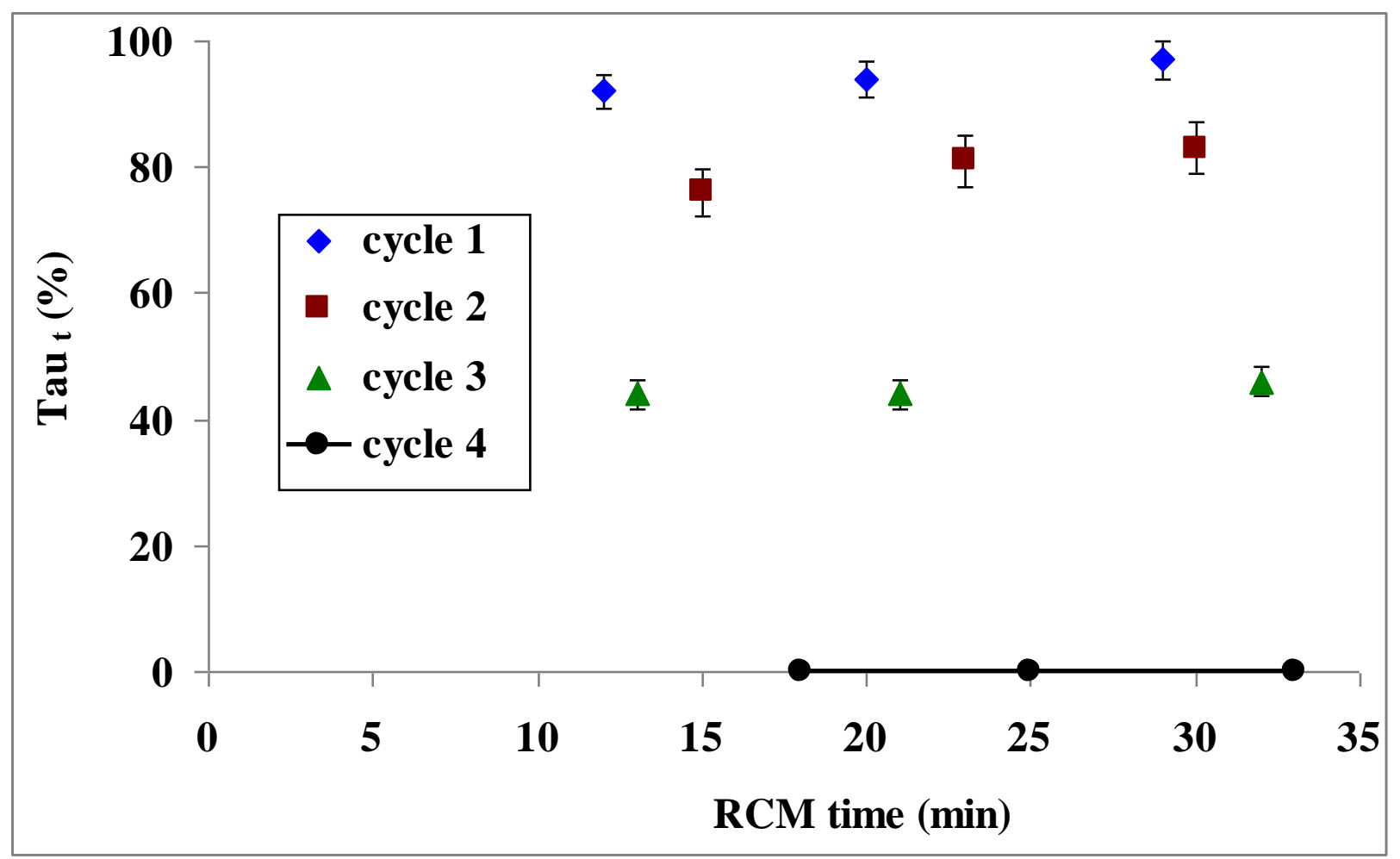

Figure 10: Evolution of DEDAM conversion $\left(\mathrm{Tau}_{t}\right)$ versus reaction time of $R C M$ at $26^{\circ} \mathrm{C}$ in toluene according to the RCM cycle. A single load of pre-catalyst at $10^{-3}$ mol. $L^{-1}$ Each load of DEDAM was $0.032 \mathrm{~mol}\left(0.106 \mathrm{~mol} . \mathrm{L}^{-1}\right)$. One load of DEDAM per RCM cycle + an OSN step up to VRR $\approx 3$ at the end of each RCM cycle. 


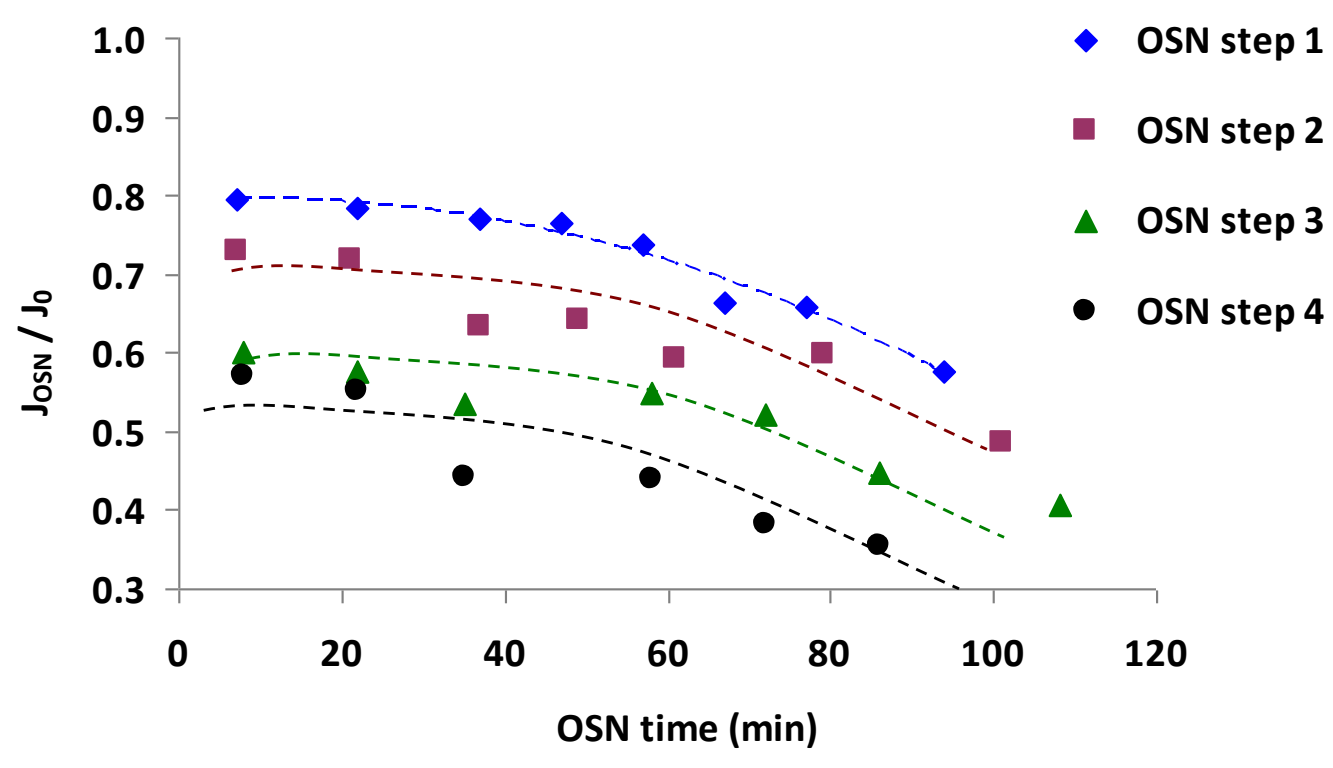

Figure 11. Evolution of the flux reduction during OSN steps of the semi-continuous membrane reactor. A single load of pre-catalyst at $10^{-3} \mathrm{~mol} . \mathrm{L}^{-1}$.

Each load of DEDAM was $0.032 \mathrm{~mol}\left(0.106 \mathrm{~mol} . \mathrm{L}^{-1}\right)$. One load of DEDAM per RCM cycle + an OSN step at 40 bar up to $V R R \approx 3$ at the end of each RCM cycle 


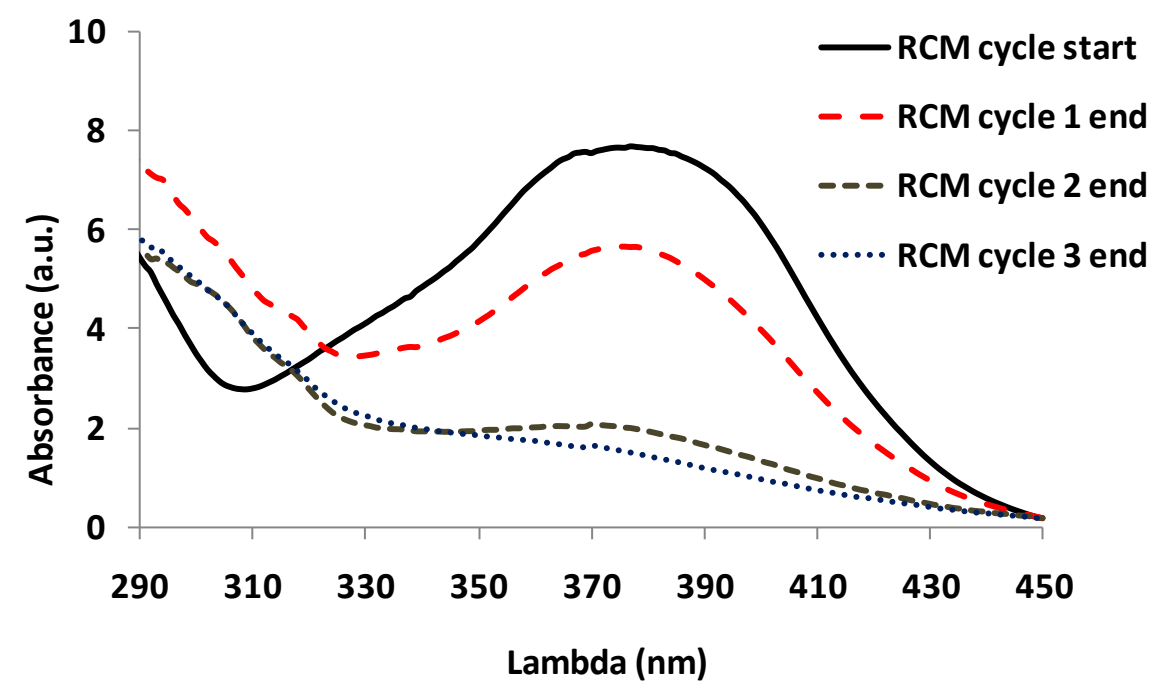

Figure 12: Evolution of $U V$-Vis spectra of retentate during $R C M$ cycles performed with $10^{-3}$ mol. $L^{-1}$ initial concentration of Grubbs-Hoveyda II pre-catalyst. Maximum absorption of the pre-catalyst form was measured at $\lambda_{\max }=377 \mathrm{~nm}$. (each spectrum was first acquired after appropriate dilution by toluene to have a maximum absorbance lower than 1 absorbance unit then the dilution correction was done to be able to directly compare the different media). 

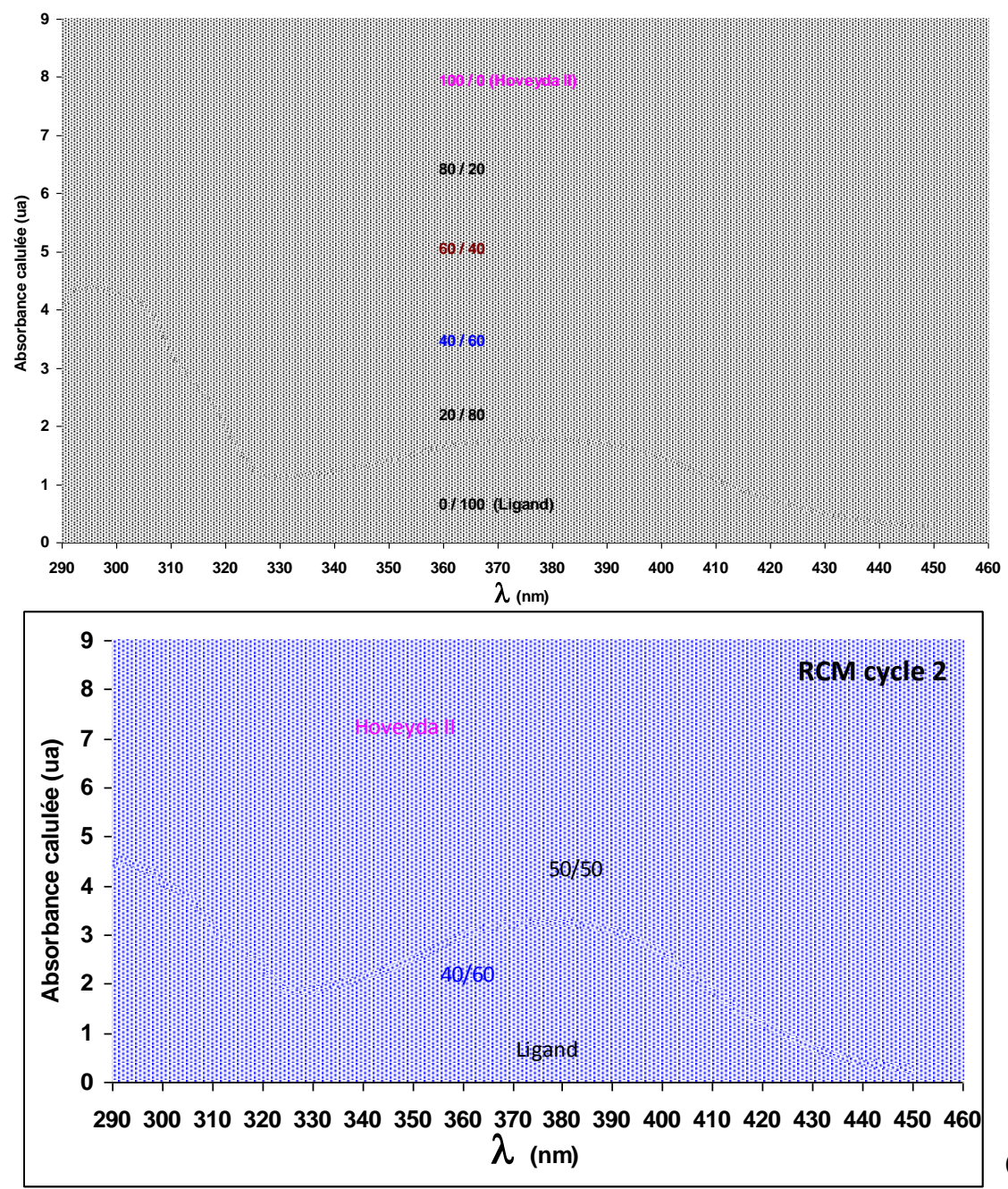

(a)

(b)

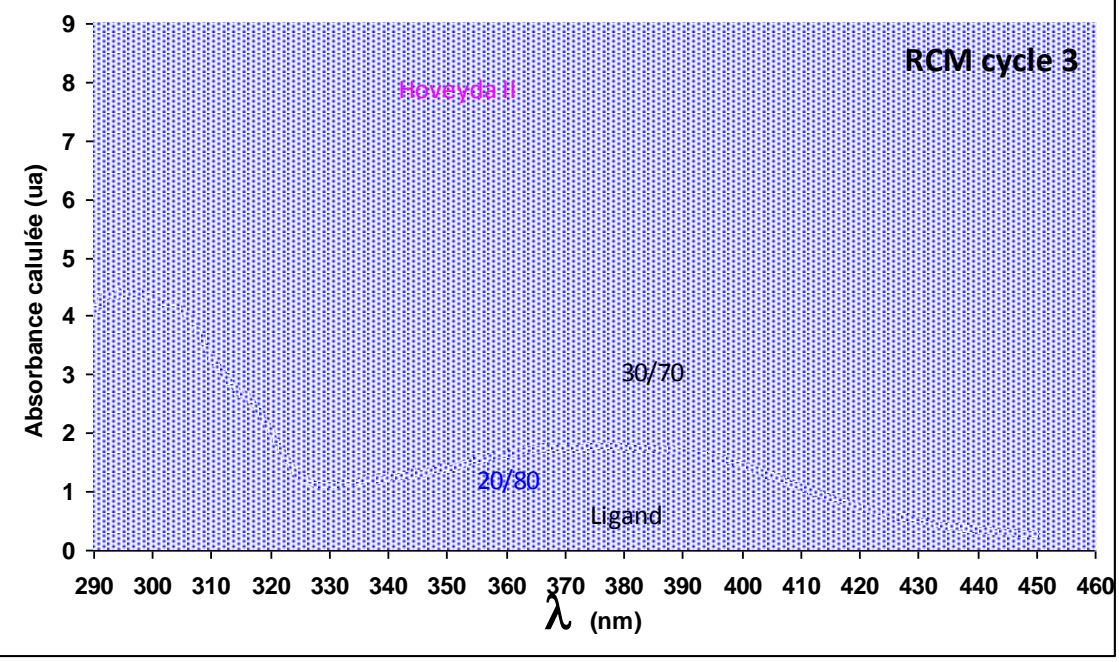

(c)

Figure 13: UV-Vis spectra of RCM media, Grubbs-Hoveyda II pre-catalyst, free isopropoxystyrene ligand and theoretical calculated spectra of mixtures of the two previous ones. (a) Experimental spectra of Grubbs-Hoveyda II and free isopropoxystyrene ligand, both at $10^{-3} \mathrm{mol.L^{-1 }}$, and theoretical calculated spectra of mixtures of the two previous ones (pre-catalyst/ligand) for an overall concentration of $10^{-3}$ mol. $L^{-1 .}(\boldsymbol{b})$ Comparison of spectrum of RCM medium at the end of RCM cycle 2 with selected theoretical spectra; (c) Comparison of spectrum of RCM medium at the end of RCM cycle 3 with selected theoretical spectra. 


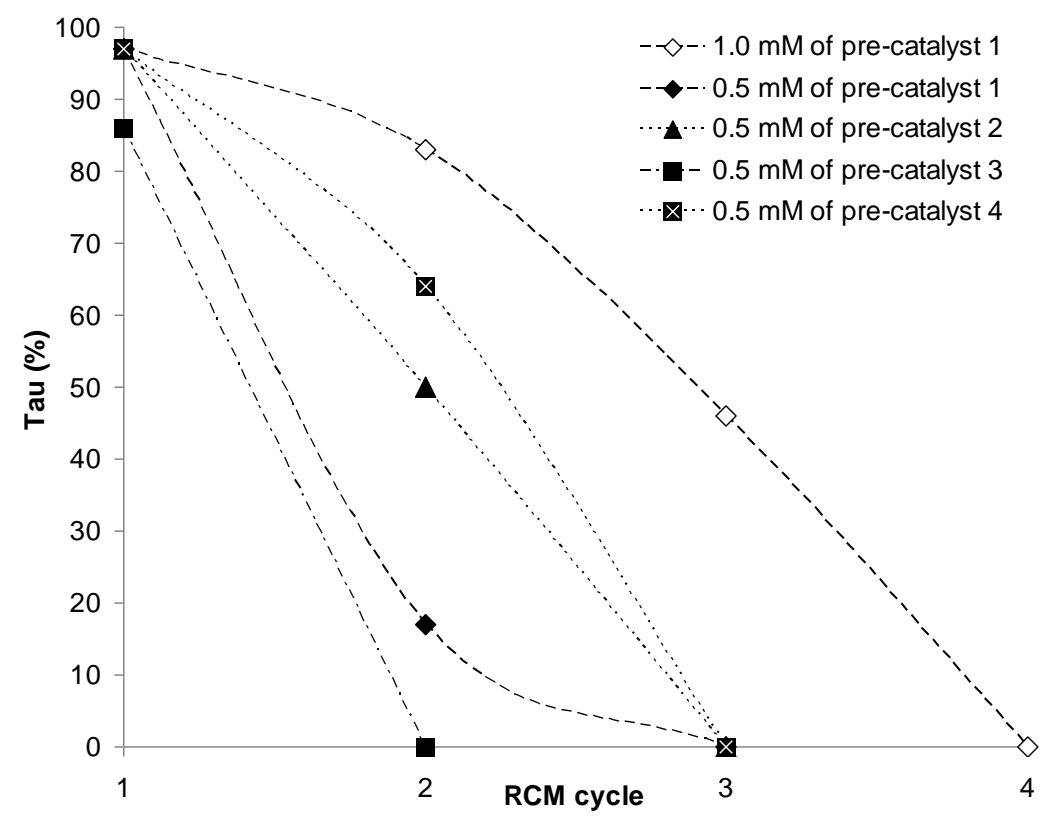

Figure 14: Maximum DEDAM conversion ratio (Tau) versus RCM cycle in the semi-continuous membrane reactor. A single load of pre-catalyst at either $10^{-3} \mathrm{~mol} . \mathrm{L}^{-1 .}$ or $0.5 \times 10^{-3} \mathrm{~mol} \cdot \mathrm{L}^{-1 .}$ Each load of DEDAM was $0.032 \mathrm{~mol}\left(0.106 \mathrm{~mol} . \mathrm{L}^{-1}\right)$. One load of DEDAM per RCM cycle + a final OSN step up to $V R R \approx 3$ at the end of each RCM cycle. 


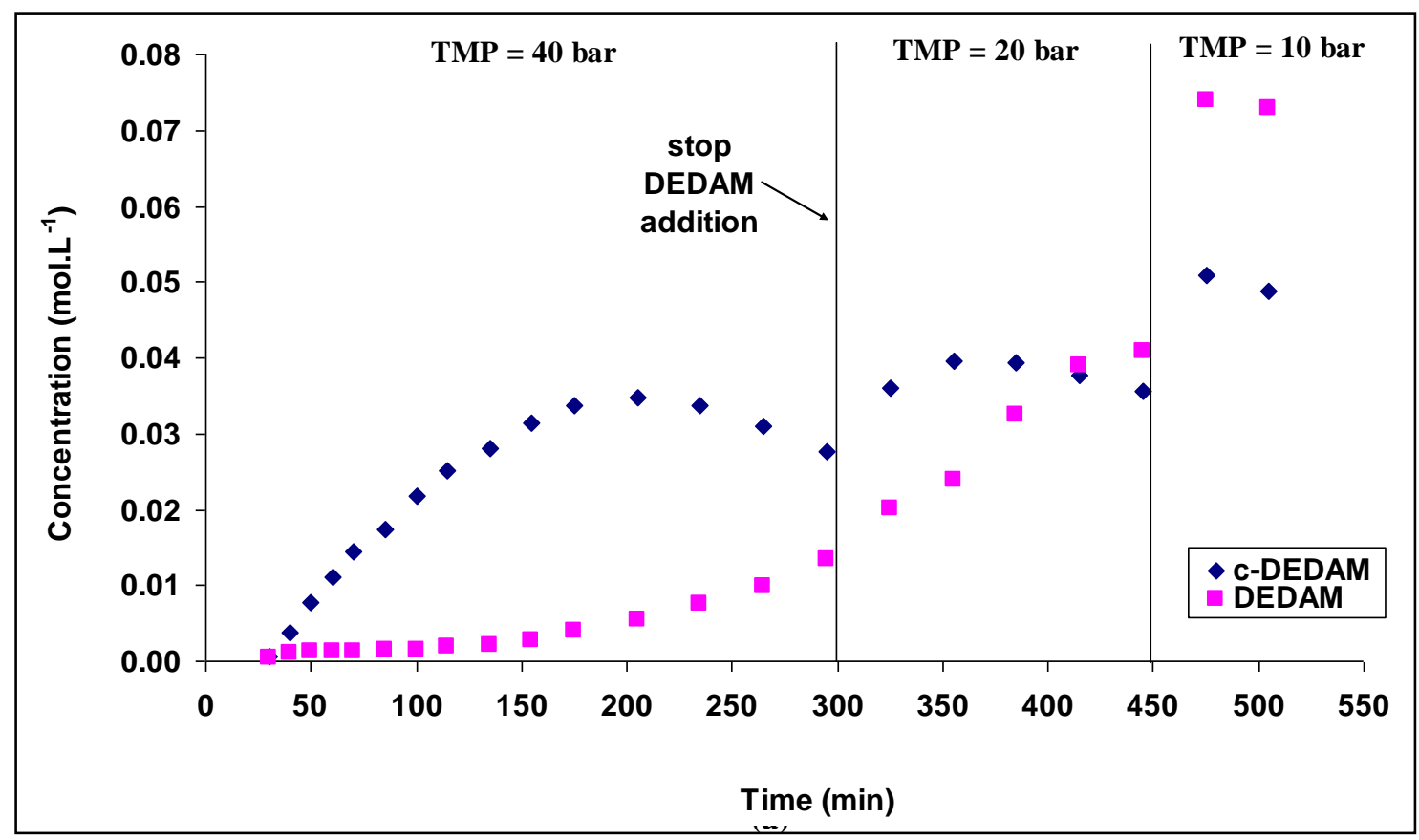

(a)

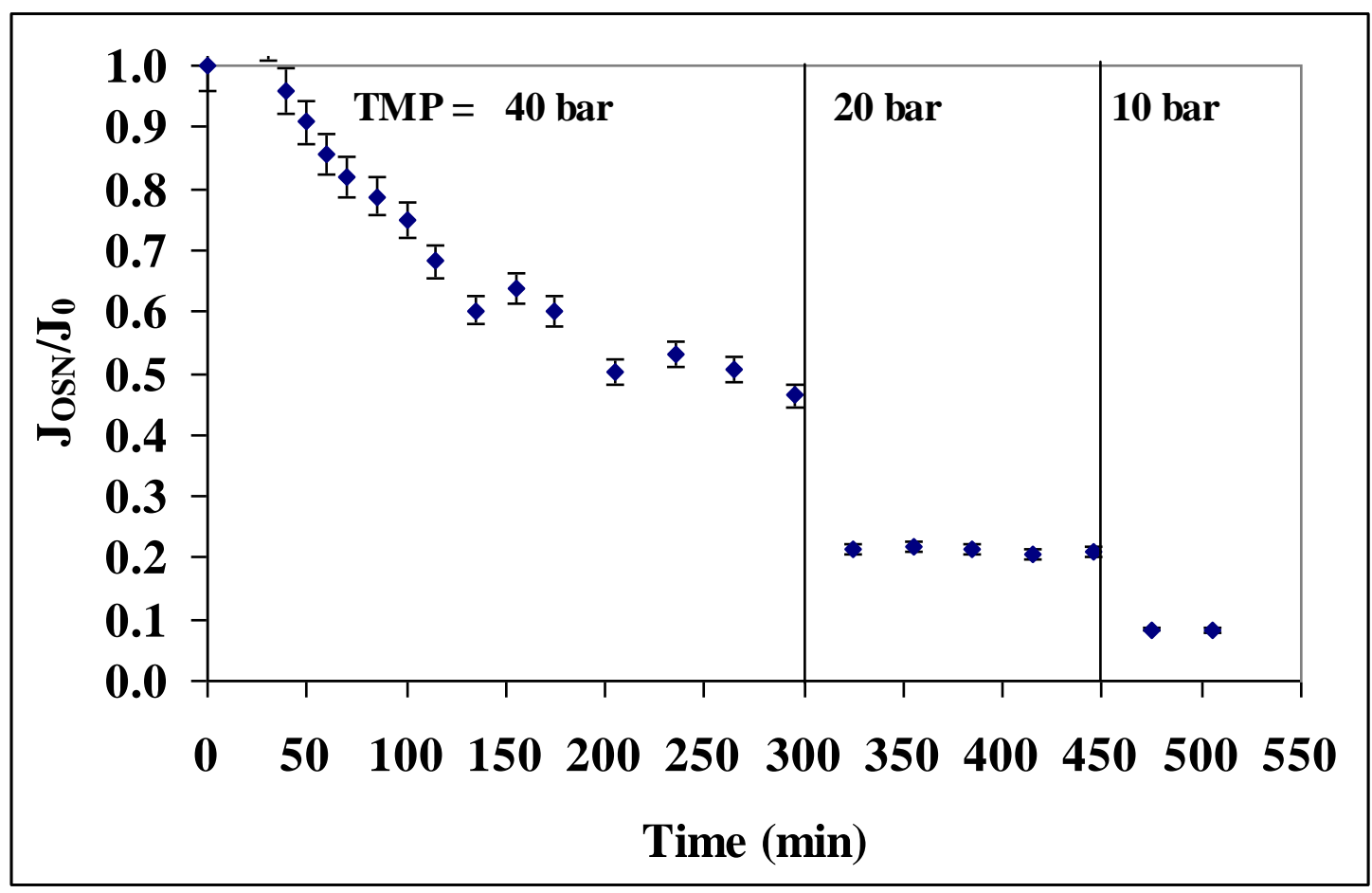

(b)

Figure 15: RCM performed in the membrane reactor running in continuous mode (a) evolution of the concentration of DEDAM and c-DEDAM in the permeate (b) evolution of the flux decrease

During DEDAM addition a 40 bar TMP was applied then TMP was decreased to enhance the product recovery. The pre-catalyst initial load was $1.6 \times 10^{-4}$ mol. The overall load of DEDAM was $0.032 \mathrm{~mol}$. Conditions: $32 \pm 4{ }^{\circ} \mathrm{C}$, cross flow velocity $\approx 0.1 \mathrm{~m} . \mathrm{s}^{-1}$. 


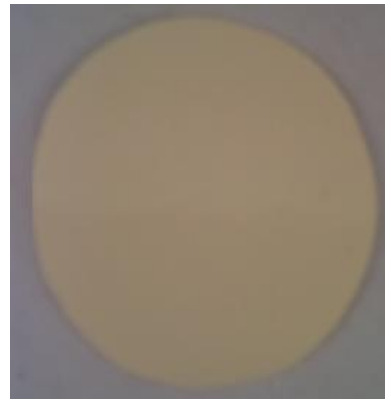

(a)

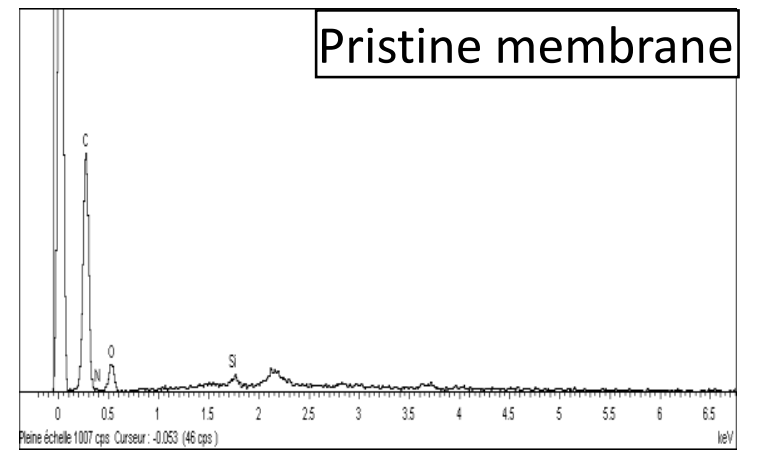

(192)

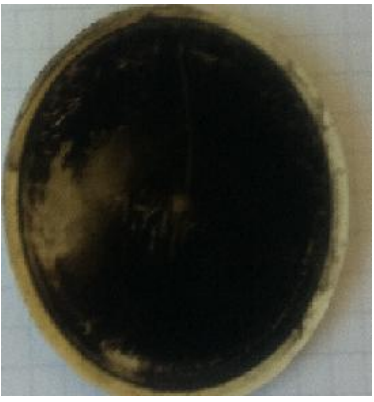

(b)

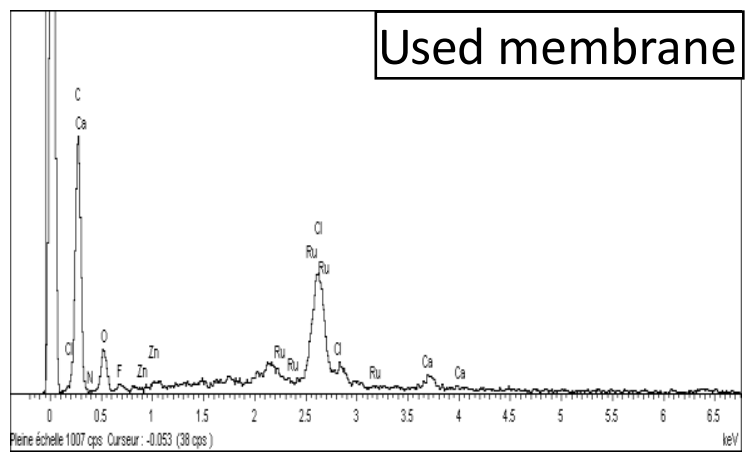

(c)

Figure 16: Pictures of (a) the pristine Starmem 122 membrane and (b) the Starmem 122 membrane after the RCM reaction at 40 bar in the continuous membrane reactor

(c) SEM-EDX analyses of the two membranes. 


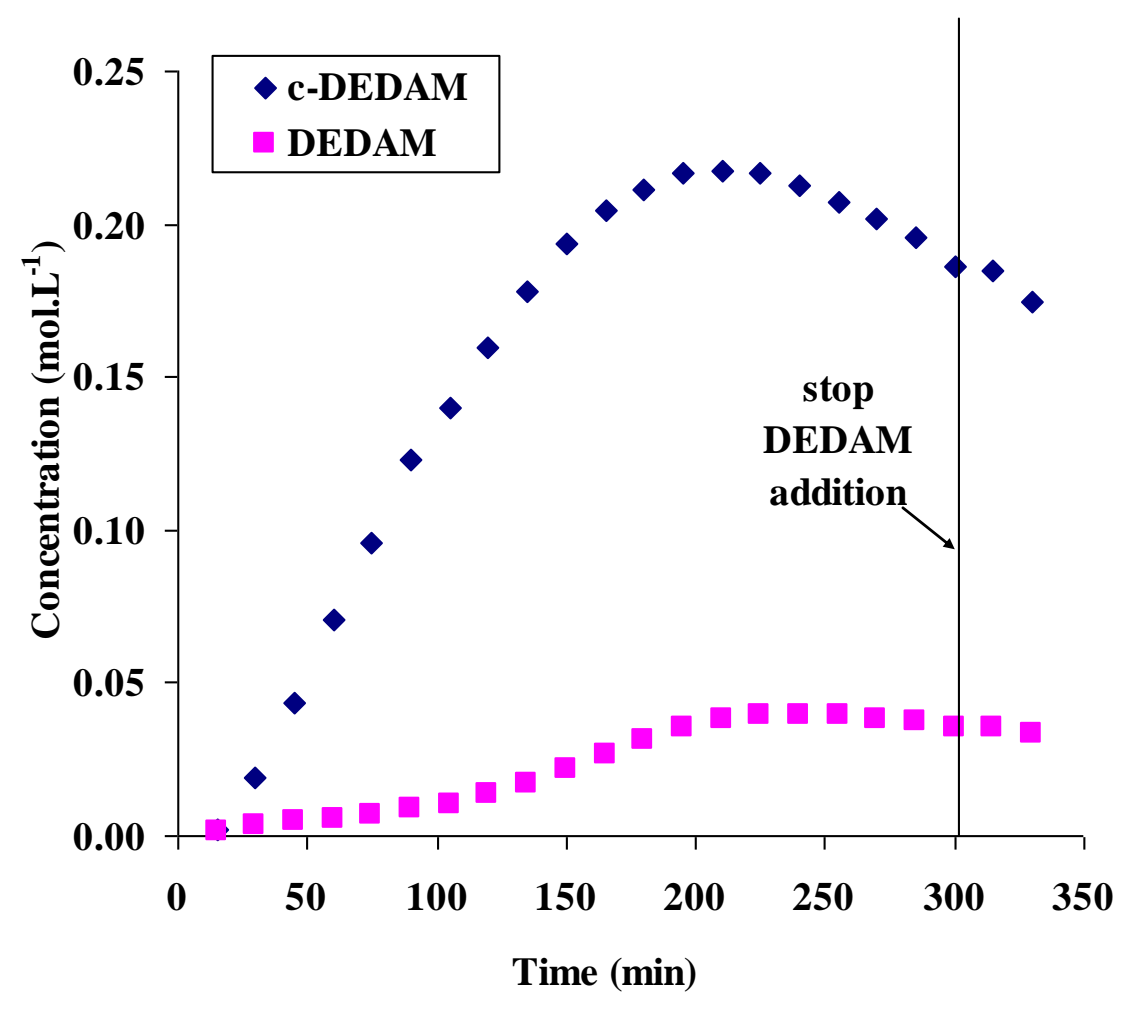

(a)

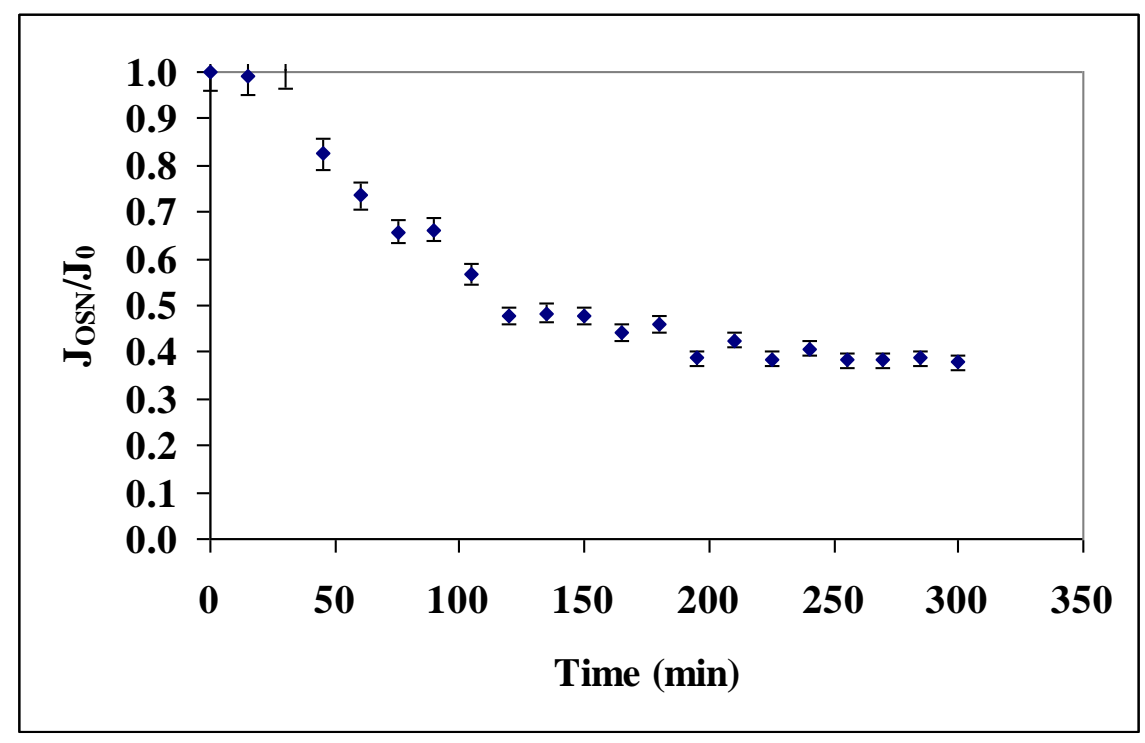

(b)

Figure 17: RCM performed in the membrane reactor running in continuous mode at 10 bar

(b) evolution of the concentration of DEDAM and c-DEDAM in the permeate (b) evolution of the flux decrease

pre-catalyst initial load was $1.6 \times 10^{-4} \mathrm{~mol}$. overall load of DEDAM was $0.032 \mathrm{~mol}$. Conditions: $32 \pm 4{ }^{\circ} \mathrm{C}$, cross flow velocity $\approx 0.1 \mathrm{~m} \cdot \mathrm{s}^{-1}$. 